ARTICLE

\title{
Distinct mechanisms orchestrate the contra- polarity of IRK and KOIN, two LRR-receptor-kinases controlling root cell division
}

\author{
Cecilia Rodriguez-Furlan (1) ${ }^{1}$, Roya Campos $^{1}$, Jessica N. Toth (1) ${ }^{1} \&$ Jaimie M. Van Norman (1) ${ }^{1 凶}$
}

In plants, cell polarity plays key roles in coordinating developmental processes. Despite the characterization of several polarly localized plasma membrane proteins, the mechanisms connecting protein dynamics with cellular functions often remain unclear. Here, we introduce a polarized receptor, KOIN, that restricts cell divisions in the Arabidopsis root meristem. In the endodermis, KOIN polarity is opposite to IRK, a receptor that represses endodermal cell divisions. Their contra-polar localization facilitates dissection of polarity mechanisms and the links between polarity and function. We find that IRK and KOIN are recognized, sorted, and secreted through distinct pathways. IRK extracellular domains determine its polarity and partially rescue the mutant phenotype, whereas KOIN's extracellular domains are insufficient for polar sorting and function. Endodermal expression of an IRK/KOIN chimera generates non-cell-autonomous misregulation of root cell divisions that impacts patterning. Altogether, we reveal two contrasting mechanisms determining these receptors' polarity and link their polarity to cell divisions in root tissue patterning.

\footnotetext{
${ }^{1}$ Department of Botany and Plant Sciences, Center for Plant Cell Biology, Institute of Integrative Genome Biology, University of California, Riverside, Riverside,

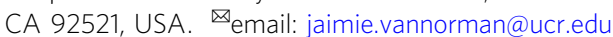


A symmetric protein distribution along different plasma membrane (PM) domains can be guided by external cues from adjacent or distal cells and may define polar axes of a tissue or organ. This coordinated polarity facilitates oriented cell division, developmental patterning, cell growth, long-range signal transduction, and the transport of ions and other molecules ${ }^{1}$. Intrinsic cellular machinery generates polar protein distribution, but the process remains poorly understood ${ }^{2,3}$. However, research has been greatly aided by examining PM proteins with contrasting polar distribution ${ }^{1}$.

A well-studied example of proteins with asymmetric distribution at the PM is the PIN-FORMED (PIN) family of auxin efflux carriers that drive directional auxin flow, which is essential for root growth and development. When expressed under their endogenous promoters, PIN1 and PIN2 exhibit contrasting polar distribution. PIN1 accumulates at the rootward PM domain in vascular and endodermal cells, while PIN2 is located at the shootward domain in epidermal and cortex cells. PIN1 polar distribution changes in different cell types indicating cell typespecific localization mechanisms ${ }^{4}$. PIN2 rootward localization in cortex cells near the stem cell niche becomes shootward as cells mature, indicating its polarity is associated with developmental context ${ }^{4}$. PIN polarity is established after cytokinesis through distinct trafficking routes for shootward/rootward localization ${ }^{4-7}$. Once established, maintenance of PIN polarity occurs by hyperpolar secretion, endocytosis and recycling, restriction of lateral diffusion, and protein phosphorylation ${ }^{8}$. However, the sorting signals and regulatory mechanisms targeting these proteins in different cell types and developmental contexts are still being investigated.

Another well-studied, contrasting protein duo is the laterally localized boric acid channel NODULIN INTRINSIC PROTEIN $5 ; 1$ (NIP5;1) and the boric acid/borate exporter REQUIRES HIGH BORON 1 (BOR1) ${ }^{9}$. Contra-polar localization of BOR1 at the inner PM domain toward the stele and NIP5;1 at the outer domain away from the stele, is proposed to be determined by cues originating from the stele ${ }^{10}$. This polar distribution is maintained in different tissues indicating an organ-level, stele-oriented polar axis enabling directional boron transport. Under boron limiting conditions, BOR1 and NIP5;1 polar distribution in epidermal and cortical cells is regulated by targeted secretion of newly synthesized proteins, constant endocytosis and recycling, cytoskeleton components, and control of lateral diffusion $3,9,11-14$. However, in endodermal cells, once BOR1 and NIP5;1 are polarly secreted, maintenance of lateral polarity is independent of the actin or microtubule cytoskeleton ${ }^{10}$. Additionally, the polarity of NIP5;1, but not BOR1, depends on phosphoinsitol synthesis ${ }^{10}$. This suggests differential mechanisms for protein accumulation to the inner or outer PM domains in endodermal cells, yet these underlying mechanisms remain poorly understood.

The leucine-rich repeat receptor-like kinase (LRR-RLK) INFLORESCENCE AND ROOT APICES RECEPTOR KINASE (IRK) exhibits polar accumulation to distinct PM domains in different cell types ${ }^{15}$. It localizes to the outer PM domain in the endodermis and pericycle and the inner domain in epidermal and cortical cells, with its polar distribution informed locally by adjacent cells. IRK is downstream of SHORT ROOT (SHR) transcriptional regulation and SHR together with the transcription factor SCARECROW (SCR) control expression of a D-type cyclin, CYCD6;1, restricting it to the cortex/endodermal initial (CEI) and the CEI daughter (CEID) leading to formative cell divisions that eventually generate endodermal and cortical cells. Strict regulation of gene expression in these cell types prevents the formation of additional ground tissue (GT) layers and maintains radial patterning ${ }^{16-20}$. CYCD6;1 promoter activity is altered in irk endodermal cells, which exhibit excess periclinal and longitudinal anticlinal cell divisions (LADs), leading to radial enlargement of the root. These phenotypes are rescued by endodermal IRK expression suggesting its polar distribution is functionally linked to repression of cell division in these cells upon a perception of extracellular cues ${ }^{15}$. However, the mechanistic basis for the establishment and maintenance of IRK polarity remains unknown.

Here, we introduce another LRR-RLK downstream of the SHR transcriptional network that, in contrast to IRK, is polarized to the inner PM domain regardless of cell type; therefore, we named it KINASE ON THE INSIDE (KOIN). The contrasting polarization of IRK and KOIN makes them a valuable pair to investigate the underlying mechanisms of LRR-RLK polarity. Our study reveals that polarization of IRK and KOIN relies mainly on the secretion of newly synthesized protein through distinct endomembrane trafficking routes. IRK and KOIN differentially accumulate during endodermal cell divisions, revealing targeted secretion to newly formed PMs. Using chimeric proteins and truncations, we found the protein domains necessary for IRK polar sorting are distinct from those of KOIN. Our results reveal coordination between LRR-RLK polarity and function to repress root cell division in the radial and longitudinal axes, adding to the growing body of work showing that polarized signal perception controls specific developmental events.

\section{Results}

KOIN and IRK show contrasting polar localization in the root. Analysis of genes expressed downstream of SHR activation in the GT led to the identification of IRK and another LRR-RLK we named KOIN (encoded by At5g58300), as candidate proteins involved in the regulation of GT cell divisions ${ }^{15,20-22}$. koin alleles have a larger root meristem with increased stele area and more numerous cells in the longitudinal axis, as measured by cortex cell number and T-junction length, phenotypes consistent with excessive cell divisions (Fig. 1a-c; Supplementary Fig. 1). Although koin roots are larger than WT roots in the radial axis, we consistently observe the expected eight GT cells around the stele and no increase in overall root length (Supplementary Fig. 1f-h). Thus, IRK and KOIN both function to repress root cell proliferation but IRK restrict GT cell number in the radial axis, while KOIN operates in the longitudinal axis.

KOIN-GFP expressed from its endogenous promoter $(K O I N p)$ was detected in the root meristem in the endodermis, phloem pole pericycle (excluded from the xylem pole pericycle), and vascular tissues (Fig. 1d, e), but not detected in the outermost cell layers. Strikingly, in the endodermis and pericycle, KOIN-GFP accumulates asymmetrically at the inner PM domain, towards the stele, which is the opposite of IRK (Fig. 1d, e, h). This KOIN-GFP reporter rescues all the observed koin mutant root phenotypes (Fig. 1g; Supplementary Fig. 2). This indicates that KOIN is a polarized LRR-RLK whose activity interior to the cortex is sufficient to modulate cell division activity across the root cell layers. Interestingly, KOIN-GFP expressed from SCRp is polarized and largely rescues the koin root phenotypes (Fig. 1g; Supplementary Fig. 3). Therefore, KOIN activity in the endodermis is sufficient to restrict cell division across root cell types and control overall meristem size. In a similar situation SCRp driven IRK-GFP expression rescues irk root phenotypes ${ }^{15}$. Thus, polarized KOIN and IRK at the endodermis are functionally important for their activities downstream of SHR.

As both, KOIN and IRK, are polarized, we tested if similar orienting cues drive their localization in different cell types. IRK localizes to distinct polar domains in various root cell types and this polarity is oriented locally by cues from adjacent cell types, particularly in the GT (Supplementary Fig. $4 \mathrm{a}, \mathrm{d}$ ) ${ }^{15}$. In contrast, 


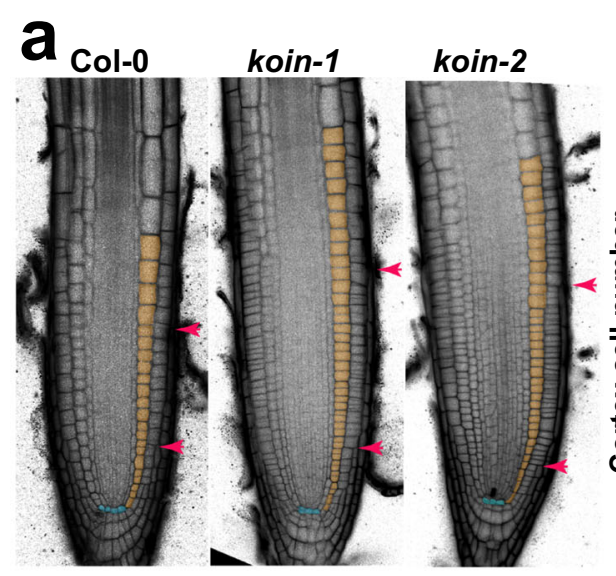

b

Col-0 koin-2 koin-1

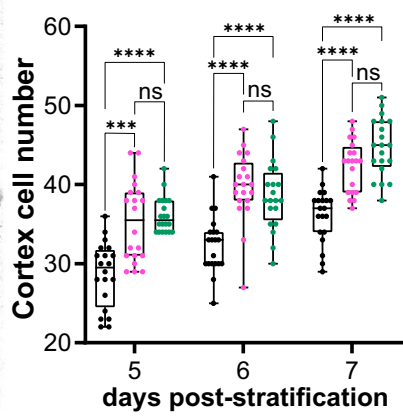

C

- Col-0 koin-2 koin-1
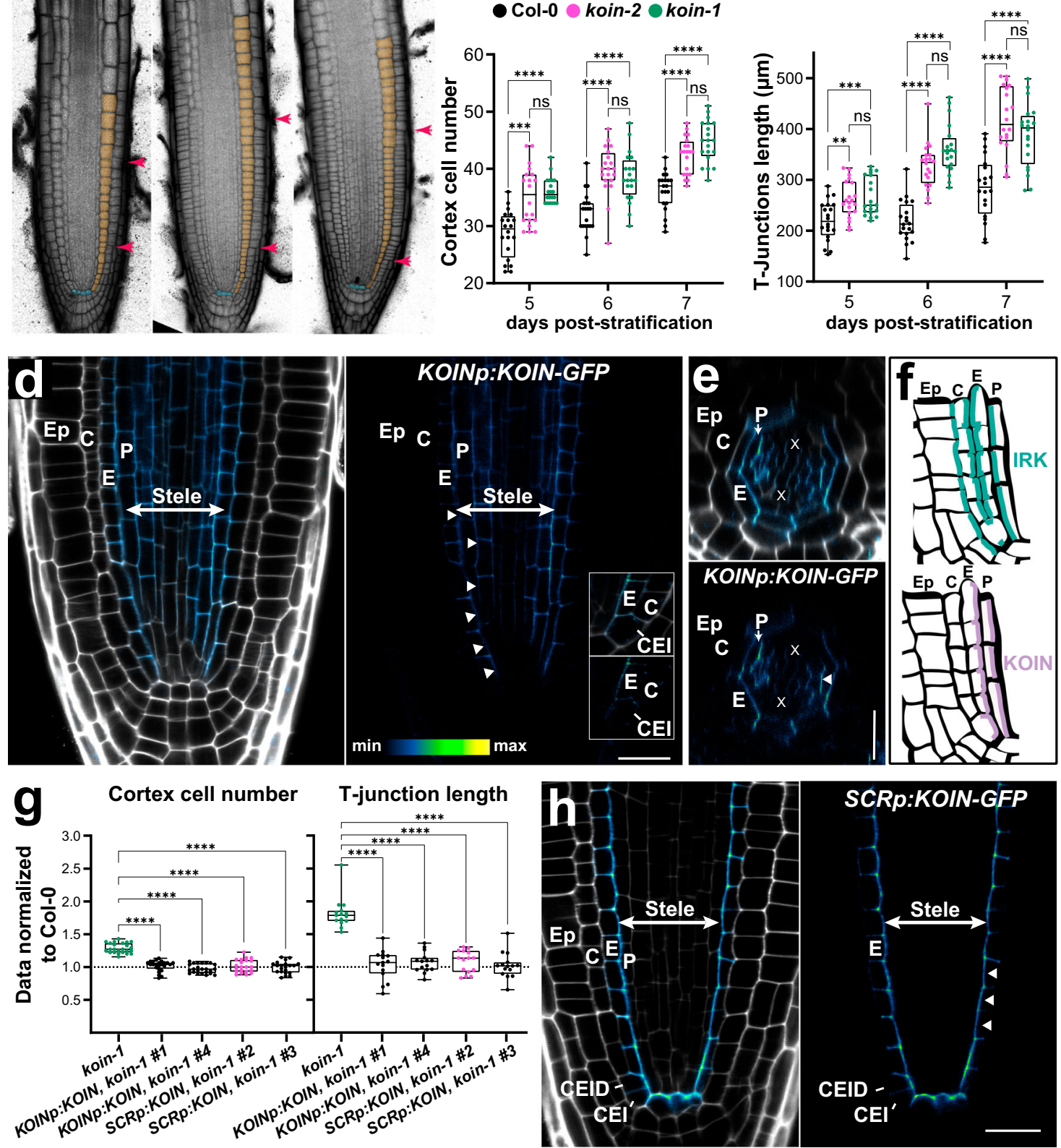

Fig. 1 KOIN functions to restrict root meristem size and is localized to the inner lateral domain in endodermal cells. a Confocal images of the median longitudinal sections of root tips with cells highlighted: quiescent center (QC, cyan), meristematic cortex (orange), and T-junctions (pink arrowheads). Quantification of $\mathbf{b}$ cortex cell number and $\mathbf{c}$ distance to the uppermost T-junction over time 5-7 days post-stratification (d.p.s.). Box plots show data from a single biological replicate representative of three with similar results $(n=20$ per genotype). Boxes delimit upper and lower quartiles, median values are indicated with a line, and single values are shown as dots. Bars indicate max/min values and ${ }^{\star \star \star *}$ statistical significance $P$ value $<0.001$ (two-way ANOVA using Turkey's multiple comparison test). d, e Confocal images of WT root meristems at 6 d.p.s. Adjacent panels show merged images of green fluorescent protein (GFP, fluorescence intensity color scale) and stained with propidium iodide (PI, gray scale) followed by GFP alone. $\mathbf{d}$ Median longitudinal and e transverse sections showing accumulation of KOIN-GFP driven by KOINp. Inset in (d) KOIN-GFP accumulation in the CEl. f Schematics summarizing endogenously expressed IRK-GFP localization (green) and KOIN-GFP (purple). $\mathbf{g}$ Quantification of cortex cell number and distance to the uppermost T-junction at 7 d.p.s. in koin-1 expressing KOIN-GFP driven by KOINp or SCRp normalized to Col-O. Data from $n=20$ per genotype for a single biological replicate representative of 3 with similar results. Boxes delimit upper and lower quartiles, median values are indicated with a line, and single values are shown as dots. Bars indicate max/min values and ${ }^{\star \star * \star}$ statistical significance $P$ value $<0.001$ (one-way ANOVA using Dunnett's multiple comparisons test). $\mathbf{h}$ Median longitudinal sections of WT roots with KOIN-GFP driven by SCRp. Cellular abbreviations: CEI cortex/endodermal initial, CEID CEI daughter, E endodermis, $\mathrm{C}$ cortex, Ep epidermis, $\mathrm{X}$ xylem axis, P pericycle. White arrowheads highlight KOIN-GFP localization to the inner lateral domain in the endodermis. Images displayed in this figure are representative of observations in experiments replicated at least 3 times with each replicate containing at least $\geq 15$ plants/genotype. Scale bars: a $50 \mu \mathrm{m} ; \mathbf{d}, \mathbf{e}, \mathbf{g} 20 \mu \mathrm{m}$. 
cell layer-specific expression of KOIN-GFP showed accumulation at the inner PM domain (stele-oriented) regardless of cell type (Fig. 1h, Supplementary Fig. 4a-g). This localization held true even in $s c r-4$, which only has one GT cell layer with mixed endodermal and cortex identity (Supplementary Fig. 4d, h). These results indicate that, unlike IRK, KOIN polarity is inner/ stele-oriented, regardless of cell type, suggesting orientation by a stele-derived cue. Therefore, different mechanisms determine the contrasting polarity of these receptors. Importantly, this contrasting polarity allows us to investigate the underlying mechanisms of LRR-RLK polar accumulation in the root GT and to connect polar receptor distribution to specific cell division control in the root.

IRK and KOIN are differentially targeted to new PMs in dividing cells. During proliferative cell divisions, PIN2 is delivered to both sides of the new PMs by non-targeted secretion followed by post-cytokinetic (re-)establishment of shootward polarity in daughter cells ${ }^{6,7}$. To determine if this mechanism applies to KOIN and IRK, we examined their polarization during proliferative, transverse anticlinal divisions (Fig. 2a). As IRK and KOIN contrapolar endodermal accumulation are central for their root meristem functions, we examined SCRp driven IRK-GFP and KOIN-mCherry $(\mathrm{mCh})$ in the same plants. IRK accumulation at newly formed PMs is detected first (Fig. 2a-e) with IRK-GFP fluorescence becoming progressively more intense towards the outer face and dimmer towards the inner face (Fig. 2a-e; Supplementary Fig. $5 \mathrm{a}-\mathrm{c}$ ). Then, KOIN becomes visible and is later detectable across new PMs with a stronger signal toward the inner endodermal face (Fig. 2f-i; Supplementary Fig. 5e, f). Therefore, during transverse anticlinal, proliferative endodermal cell divisions IRK and KOIN secretion is spatiotemporally targeted to new PMs, while lateral polarity remains undisturbed.

Unlike proliferative divisions, during formative asymmetric cell divisions, cellular components, such as organelles and proteins, are unequally distributed to the daughter cells ${ }^{23}$. Therefore, we examined IRK and KOIN distribution during and after CEI/CEID formative cell divisions (Fig. 2a), as this may contribute to the establishment of endodermal lateral polarity. In the CEI, KOIN is absent only from the outer PM domain, while IRK is restricted to the center of the shootward and rootward domains (Fig. $2 \mathrm{j}$ and Supplementary Fig. $5 g-j$ ). Upon formative, transverse anticlinal division of the CEI, both KOIN and IRK are detected at new PMs (Fig. 2a, k, l). However, IRK mainly accumulates at the center of these new membranes, while KOIN is highest at the inner side and declines towards the outer side (Supplementary Fig. 5k-n). Remarkably, upon the formative periclinal division of the CEID, only IRK is detected at the new PMs (Fig. $2 \mathrm{~m}-\mathrm{o}$ ). This division plane coincides with the outer polar domain of newly formed endodermal cells and KOIN is not detectable at this domain. Subsequently, the inner/outer PM localization of KOIN and IRK is maintained as endodermal cells undergo further proliferative divisions. Therefore, the inner/outer polarity of the endodermal daughter cell is directly influenced by CEID-specific sorting of IRK and exclusion of KOIN from the new PMs as division occurs.

To gain insight into the relationship between periclinal divisions of the CEID and IRK lateral polarization we used, as a tool, pub4-1, a mutant with a greater number of CEI/CEID ${ }^{24}$. We delayed the formative division of the CEID by treating pub4-1 with the chemical Brefeldin A $(\mathrm{BFA})^{25}$. After $5 \mathrm{~h}$, and more evidently after $24 \mathrm{~h}$ treatments, undivided CEIDs were interspersed with newly formed endodermal cells (Supplementary Fig. 5o, p). Rootward/shootward IRK-GFP localization in the CEID was maintained until the CEID divided, then IRK polarization to the outer PM domain in the endodermis was established and remained unaltered after further divisions (Supplementary Fig. 5o). These results further demonstrate a link between the formative, periclinal division of the CEID and the establishment of IRK lateral polarity.

Distinct secretion pathways direct IRK and KOIN polarization. Determining KOIN-GFP and IRK-GFP dynamics may help us understand the mechanisms underlying the regulation of polarity and its relationship to protein function. Analysis of fluorescence recovery after photobleaching (FRAP) can reveal differential dynamics of polarized proteins, which may arise from protein secretion, recycling, lateral diffusion, and/or local interactions ${ }^{8,26}$. After photobleaching, faint signal recovery (less than 15\%) was observed for both KOIN-GFP and IRK-GFP, suggesting that these PM proteins have little to no lateral diffusion and that the initial pool of proteins is replaced at a slow rate (Supplementary Fig. 6a-j). Many polarized PM proteins have a low recovery rate ret, $^{3,7}$ and appear relatively stationary, yet IRK and KOIN recovery appear to be slower even among them.

To further characterize IRK and KOIN protein dynamics we used a set of chemicals that interfere with protein trafficking at distinct points in the secretory and endocytic pathways (summarized in Supplementary Table 1). BFA partially interferes with protein secretion by targeting a subset of guanidine exchange factors (GEFs) acting on ARF-GTPases (ARF-GEFs) resulting in intracellular agglomerations called BFA bodies that co-localize with trans-Golgi network (TGN) markers ${ }^{27-30}$. One-hour BFA treatment $(50 \mu \mathrm{M})$ led to intracellular IRK-GFP accumulation in BFA bodies but did not affect its polar distribution (Fig. 3a, b, e, f; Supplementary Fig. 7a). To determine whether IRK-GFP accumulation at BFA bodies corresponds to proteins trafficked after de novo synthesis and/or proteins cycling back to the PM after endocytosis (recycling), we pre-treated with the protein synthesis inhibitor cycloheximide (CHX) followed by cotreatment with BFA. IRK-GFP was depleted from BFA bodies indicating that the GFP signal corresponds to interference with trafficking of newly synthesized protein (Fig. 3a, b). Accordingly, extended CHX treatments nearly depleted IRK-GFP signal from the PM indicating that newly synthesized protein is the main contributor to IRK polar accumulation and that the pool of PM protein present prior to the treatment was degraded (Fig. 3a). In contrast, a substantial fraction of KOIN-GFP traffic is not affected by BFA, as only faint agglomerations were visible in a few pericycle cells, and which disappeared upon pre-treatment with CHX (Fig. 3c, d). CHX treatments decreased KOIN-GFP accumulation at the PM and the signal was nearly undetectable after $6 \mathrm{~h}$ pointing to degradation of the existing PM protein pool. Thus, CHX treatments indicate that the polarization of both proteins is predominantly dependent on the secretion of the newly synthesized protein. Additionally, accumulation of IRK, but not KOIN, at newly formed PMs after proliferative endodermal cell divisions was also sensitive to BFA treatment (Fig. 3e, f, n). These results show that a BFA-insensitive secretion pathway is a principal contributor to KOIN polarity.

As IRK and KOIN polar endodermal accumulation is sufficient for function, we continued to analyze mechanisms of polarization specifically in this cell type using a complementary set of compounds previously shown to affect protein polarization. Disruption of the microtubule or actin cytoskeleton did not alter the endodermal polar distribution of BOR 1 and NIP5; $1{ }^{10}$ or IRK and KOIN (Supplementary Fig. 7b, c). Additionally, no change was observed upon treatment with Wortmannin $(\mathrm{Wm})$, an inhibitor of phosphoinositol synthesis, which affects lateral polarization of NIP $5 ; 1$ in the endodermis ${ }^{10}$. However, extended Wm treatment decreased IRK and KOIN accumulation at the 
a
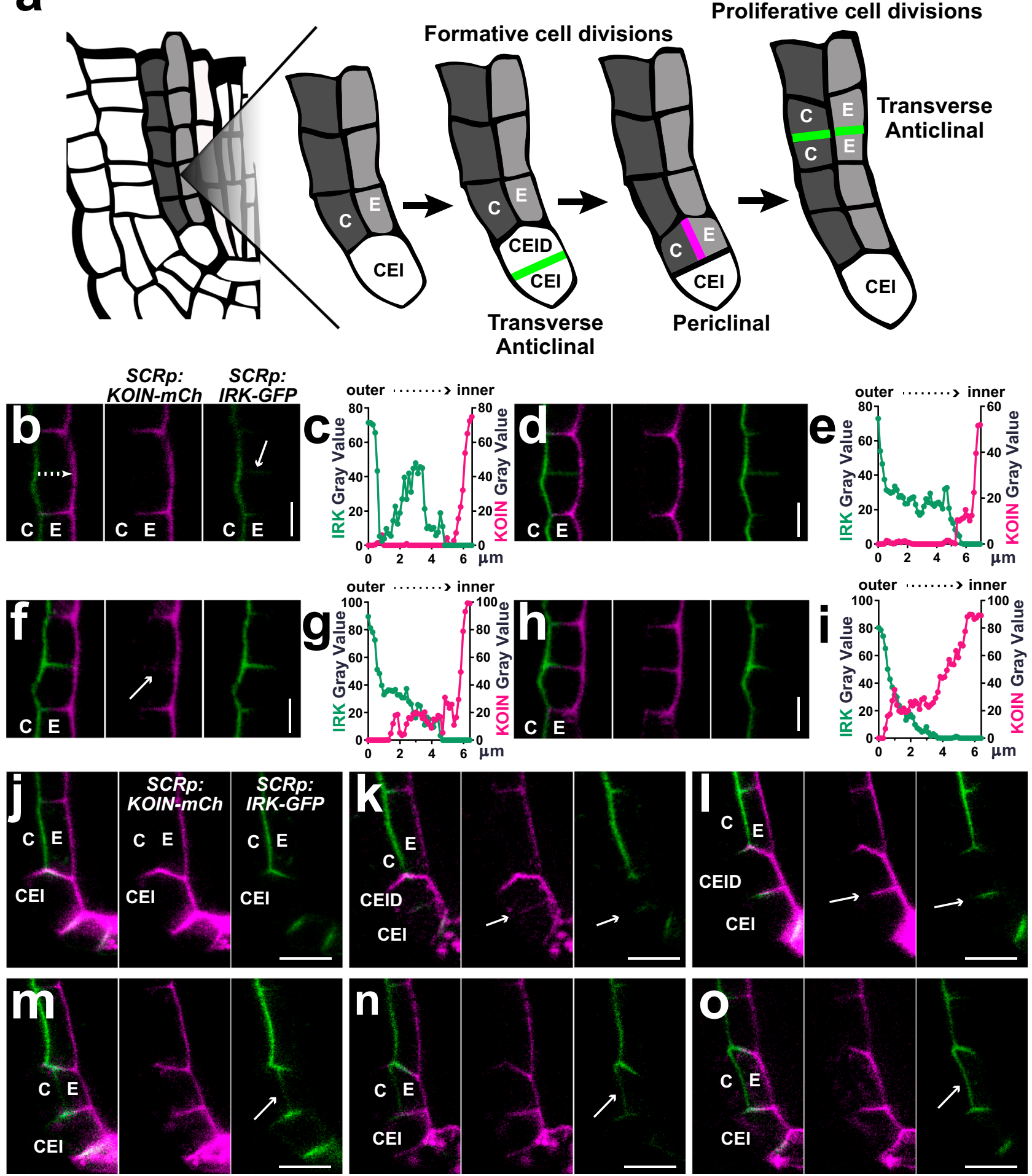

Fig. 2 Dynamic polar distribution of KOIN and IRK upon GT cell divisions. a Schematics of a portion of a WT root tip highlighting formative and proliferative cell divisions during GT development. (Left to right) Formative transverse anticlinal division of the CEl (green) followed by periclinal formative division of the CEID (pink) to form endodermal and cortex cells. GT cells then proliferate through transverse anticlinal cell divisions (green). $\mathbf{b}, \mathbf{d}, \mathbf{f}, \mathbf{h}, \mathbf{j}-\mathbf{o}$ ) Adjacent panels show KOIN-mCherry ( $\mathrm{mCh}$, magenta) and IRK-GFP (green) driven by SCRp with fluorescence images merged and then each channel alone. $\mathbf{b}, \mathbf{d}, \mathbf{f}, \mathbf{h} \mathrm{KOIN}-\mathrm{mCh}$ and IRK-GFP localization during proliferative transverse anticlinal endodermal cell divisions. $\mathbf{c}, \mathbf{e}, \mathbf{g}$, $\mathbf{i}$ Graphs of fluorescence intensity gray values (arbitrary units) across the new PM represented in $\mu$ m (dashed arrow as shown in (b): IRK-GFP (green lines) and KOIN-mCh (magenta lines). j-o KOIN-mCh and IRK-GFP localization in the CEI (j) before and ( $\mathbf{k}-\mathbf{I})$ during formative transverse anticlinal cell division and in the CEID ( $\mathbf{m}-\mathbf{o})$ during formative periclinal cell division. Note the CEl, cortex, and endodermal cells have elongated in (o). White arrows indicate fluorescence detected at new PMs. Scale bars: $10 \mu \mathrm{m}$. Images are representative of studies that were performed at least three times with at least 15 plants per condition per replicate. 


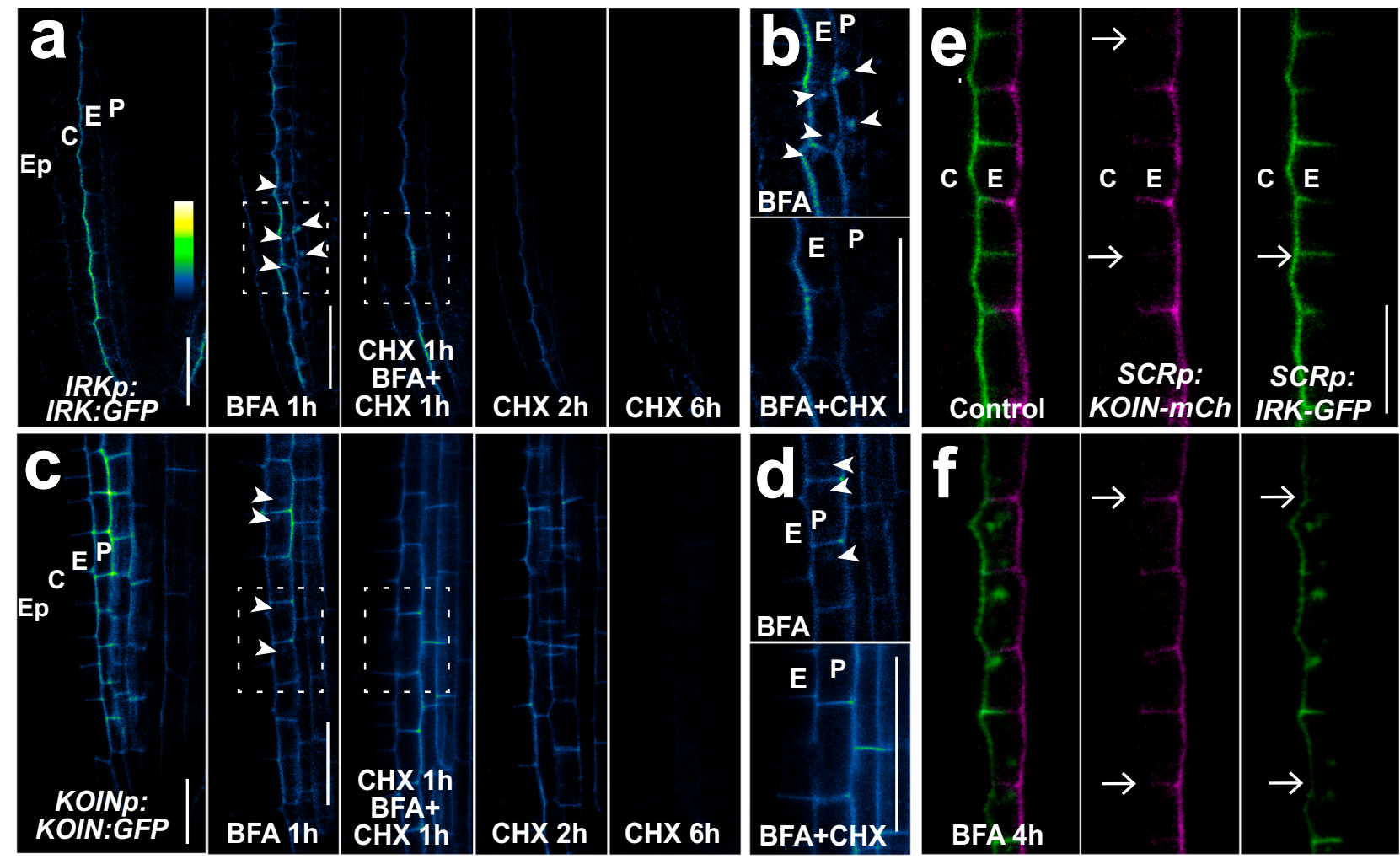

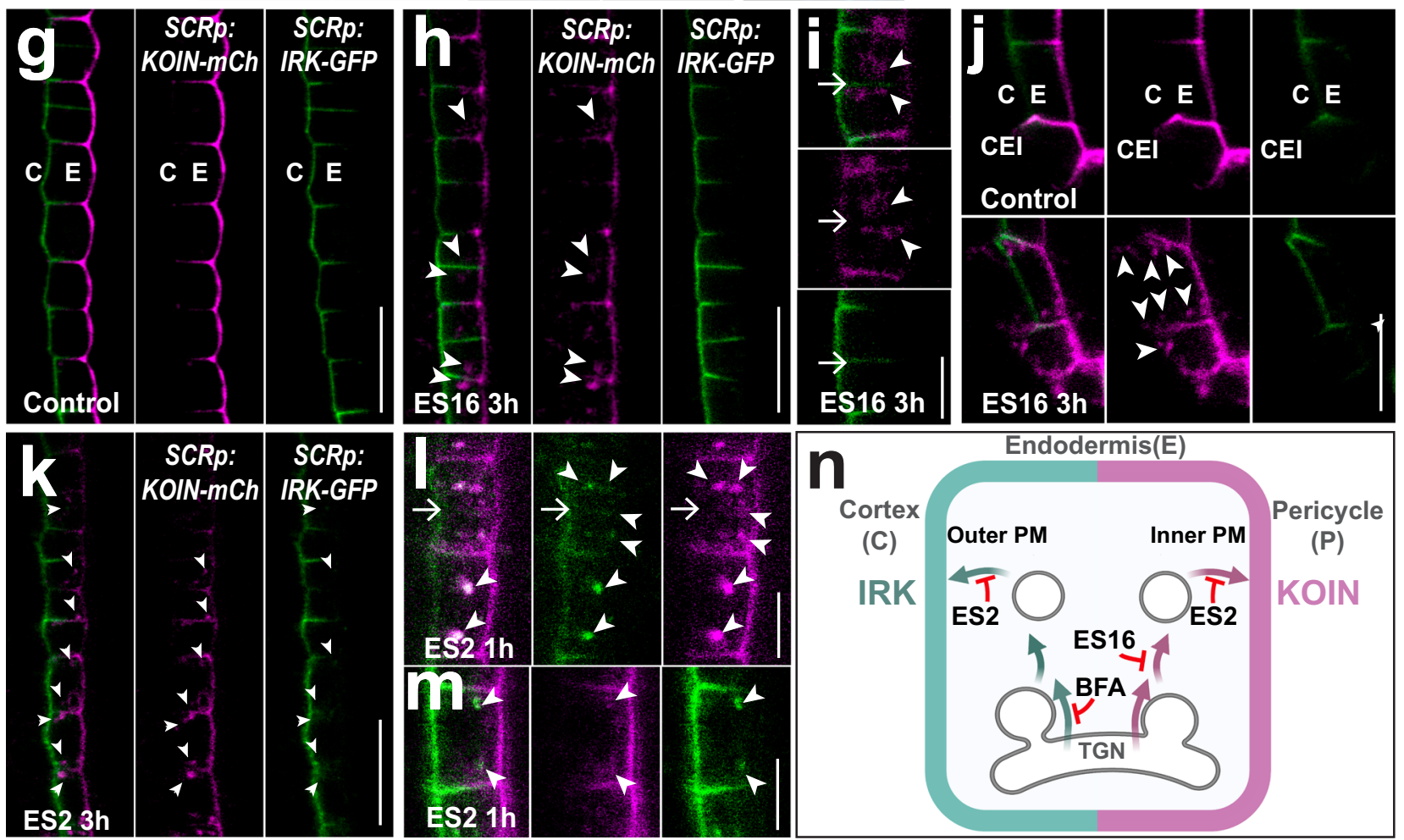

PM (Supplementary Fig. 7f, g), indicating that phosphoinositol synthesis contributes to their stability or delivery.

We next treated the roots with Endosidin16 (ES16), which alters shootward PIN2, but not rootward PIN1 secretion by targeting the RAB GTPase A2A (RABA2A) $)^{5}$. Interestingly, ES16 affected secretion of KOIN, but not IRK, to newly formed PMs during proliferative and formative cell divisions (Fig. $3 \mathrm{~g}-\mathrm{j}$ ). Thus, an ES16 sensitive mechanism is involved in the secretion of KOIN, but not of IRK, which could explain temporal differences in their accumulation at newly formed PMs (Fig. 3n). We also treated the roots with Endosidin2 (ES2), which produces intracellular PIN2 agglomerations by targeting EXO70A1 and interfering with polar secretion from the TGN to the $\mathrm{PM}^{31}$. ES2 treatment caused intracellular IRK and KOIN agglomerations, particularly near the division plane (Fig. 3k-m), suggesting targeting of both proteins to new PMs requires EXO70A1. Furthermore, extended ES2 treatment significantly reduced the overall accumulation of IRK and KOIN at the PM 
Fig. 3 Chemical treatments assessing endomembrane trafficking contributions to IRK and KOIN polar distribution. a, c Confocal images of WT roots expressing IRKp:IRK-GFP and KOINp:KOIN-GFP with GFP shown in fluorescent intensity color scale. Roots treated with DMSO (control), $50 \mu \mathrm{M}$ BFA for $1 \mathrm{~h}$ (squares indicate magnified images in (b) and (d)), $50 \mu \mathrm{M} \mathrm{CHX}$ for $1 \mathrm{~h}$ followed by a co-treatment $50 \mu \mathrm{M} \mathrm{BFA}$ and $50 \mu \mathrm{M} \mathrm{CHX}$ for an additional $1 \mathrm{~h}$, and finally, treatment with $50 \mu \mathrm{M} \mathrm{CHX}$ for 2 and $6 \mathrm{~h}$. White arrowheads indicate BFA bodies. e, f Confocal images (merged) of roots expressing IRK-GFP (green) and KOIN-mCh (magenta) from SCRp treated with (e) DMSO (control) and (f) $50 \mu \mathrm{M}$ BFA for $4 \mathrm{~h}$. White arrows indicate the differential distribution of IRK and KOIN at new PMs. $\mathbf{g}-\mathbf{i}, \mathbf{k}-\mathbf{m}$ Endodermal cells and (j) CEI expressing SCRp driven IRK-GFP (green) and KOIN-mCh (magenta) with merged images followed by single channels. $\mathbf{g}$ Control (DMSO) conditions, $\mathbf{h}$, i treated with $15 \mu \mathrm{M} \mathrm{ES16} \mathrm{for} 3 \mathrm{~h}$. Upon ES16 treatment KOIN-mCh agglomerations (arrowheads) are observed adjacent to the forming cell plate where IRK-GFP is already accumulating (white arrows). $\mathbf{j}$ CEI in control conditions or after $3 \mathrm{~h}$ treatment with $15 \mu \mathrm{M}$ ES16. White arrowheads indicate KOIN agglomerations in (h, $\mathbf{i})$ endodermal cells near the transverse anticlinal division plane and in (j) the CEI and after the periclinal division of the CEID. $\mathbf{k}-\mathbf{m}$ Endodermal cells of roots treated with $40 \mu \mathrm{M}$ ES2 for $3 \mathrm{~h}$. Arrowheads indicate ES2-induced agglomerations of IRK-GFP and KOIN-mCh. I The arrows indicate forming PMs and the arrowheads show the ES2 agglomerations. n Schematic of an endodermal cell with the cortex (left) and pericycle (right) summarizing chemical treatment results (created at BioRender.com). IRK (green) and KOIN (purple) trafficking to the outer and inner domains of the PM, respectively. IRK and KOIN are secreted from different TGN domains. IRK is secreted through a BFA- and ES2-sensitive pathway, while KOIN is secreted through an ES16- and ES2-sensitive pathway. Scale bars: $15 \mu$ m (a-h, $\mathbf{j}$, $\mathbf{k}$ ); $5 \mu \mathrm{m}(\mathbf{i}, \mathbf{I}, \mathbf{m})$. Images are representative of observations in experiments replicated at least 3 times with each containing at least 15 plants/conditions.

(Supplementary Fig. 7d, e). This depletion indicates that pools of PM-localized IRK and KOIN, present prior to ES2 treatments, were degraded. Consistent with this, ES2 was shown to enhance PIN2 endocytic traffic to the lytic vacuole ${ }^{32}$. IRK and KOIN trafficking to the lytic vacuole is also evidenced by their retention in doughnut-shaped agglomerations induced by Wm interference with degradative trafficking (Supplementary Fig. 7h). However, the Wm-induced IRK and KOIN accumulations did not always co-localize, suggesting differential timing of their transport to the vacuole.

Altogether, our results show that IRK and KOIN polarization is driven by polar secretion of newly synthesized protein through distinct pathways that confer specificity of cargo delivery to the cell division plane and/or the lateral membranes (Fig. 3n).

KOIN protein sorting and function require its kinase domain. Studies in mammalian and yeast systems indicate that several transmembrane proteins contain cytosolic sorting motifs recognized by adapter proteins that facilitate their delivery to specific destinations ${ }^{33-35}$. However, studies on polar sorting motifs in plants are less advanced ${ }^{13,14,36}$. We reasoned that polar sorting of IRK and KOIN may be determined by distinct protein-specific domains and investigated this using a molecular domain swapping approach. LRR-RLKs are composed of an extracellular domain containing multiple LRRs, a transmembrane domain (TM), and a cytoplasmic kinase domain (KD) (Fig. 4a). First, we tested if KOIN localization was disrupted by swapping the KOIN TM and kinase domains with those from IRK to generate

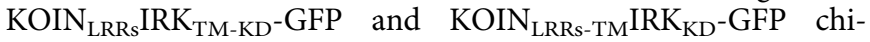
meras (Fig. 4b). When expressed under the control of the SCRp in WT both chimeras showed nonpolar localization in endodermal, CEI/CEID, and QC cells (Fig. 4c). Thus, the KOIN extracellular domain is not sufficient to direct its polarization. Consistent with this, a KOIN truncation lacking the LRR domains ( $\triangle \mathrm{LRRs}$ ) is largely retained in endomembrane compartments, but the portion that reaches the PM accumulates to the inner polar domain (Supplementary Fig. 8a-c).

To test if the KOIN cytoplasmic domains were responsible for its polar localization, we truncated KOIN removing the juxtamembrane (Jx) and KD (KOIN $\Delta \mathrm{JxKD})$, and found that it had nonpolar accumulation at the PM (Fig. 4c, d). Therefore, KOIN sorting determinants are contained in the cytoplasmic domains and, remarkably, in the absence of these domains, KOIN protein secretion lacks specificity and is misdirected with nonpolar accumulation in endodermal, CEI/CEID, and QC cells. Given its nonpolar accumulation, we also investigated whether KOIN $\Delta$ JxKD could rescue koin root phenotypes. However, endodermalexpressed KOIN $\triangle \mathrm{KD}-\mathrm{mSC}$ in koin remains nonpolar and, in contrast to full-length KOIN, fails to rescue koin root phenotypes (Supplementary Figs. 4 and 9). These results indicate that the KOIN $\mathrm{KD}$ is required for it to be actively recognized and sorted into a targeted polar secretion pathway and to function to repress root cell divisions.

IRK polar sorting determinants reside in the extracellular domain. As the KOIN intracellular domain is sufficient for its polarity, we determined if this precedent could be extended to IRK. We first fused the IRK extracellular and TM domains to the KOIN KD generating the IRK $_{\text {LRRs-TM }} \mathrm{KOIN}_{\mathrm{KD}}$-GFP chimera (Fig. 4e). Upon expression by $S C R p$ in WT, this chimera accumulates like IRK to the outer PM domain, and a similar localization was observed for the $\mathrm{IRK}_{\mathrm{LRRs}} \mathrm{KOIN}_{\mathrm{TM}-\mathrm{KD}}-\mathrm{GFP}$ chimera (Fig. 4f).

IRK and KOIN are classified as atypical or pseudo-kinases because they contain non-conserved residues at key positions in their KDs (Supplementary Fig. 10a), and they are reported to lack kinase activity $37-40$. We next determined how polar sorting was affected by the fusion of the active $\mathrm{KD}$ of the LRR-RLK ERECTA $^{41}$ to the IRK extracellular and TM domains (Supple-

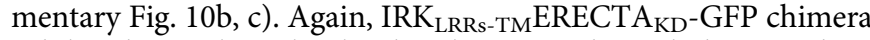
exhibited outer lateral polar distribution in the endodermis. Thus, in the endodermis, the IRK extracellular domain is the dominant determinant directing the protein to the outer polar domain, while the TM or KD of IRK are exchangeable, regardless of KD activity status.

To further dissect the contribution of the IRK extracellular domain to polarity, we performed a series of deletions of LRR domains. However, all IRK $\triangle \mathrm{LRR}$ truncations were retained at the endoplasmic reticulum (ER, Supplementary Fig. 8d-h). This indicates that the exit of IRK from the ER requires precise quality control of the structure of its extracellular domain. To continue assessing the extracellular domain's importance in determining IRK polarity, we created truncated versions lacking the KD (IRK $\triangle \mathrm{KD}-\mathrm{GFP}$ ) or the entire cytoplasmic domain (Jx and KD; IRK $\Delta \mathrm{JxKD}-\mathrm{GFP}$ ) (Fig. $4 \mathrm{~g}, \mathrm{~h}$ ). These IRK truncations retained their polar accumulation in the CEI/CEID and endodermis and when expressed under the control of CO2p or IRKp were distributed similarly to full-length IRK (Supplementary Fig. $10 \mathrm{~d}-\mathrm{g}$ ). Therefore, truncated IRK $\Delta K D$ and IRK $\Delta \mathrm{JXKD}$ are recognized and correctly sorted in different cell types. Thus, contrary to our knowledge in other systems $24,25,35$, the IRK extracellular, not the cytoplasmic domain, carries the sorting determinants for its polarity. Collectively, our evidence indicates that in plants at least two different sorting mechanisms directing LRR-RLK polarization exist, one recognizing extracellular motifs and another recognizing cytoplasmic motif. 

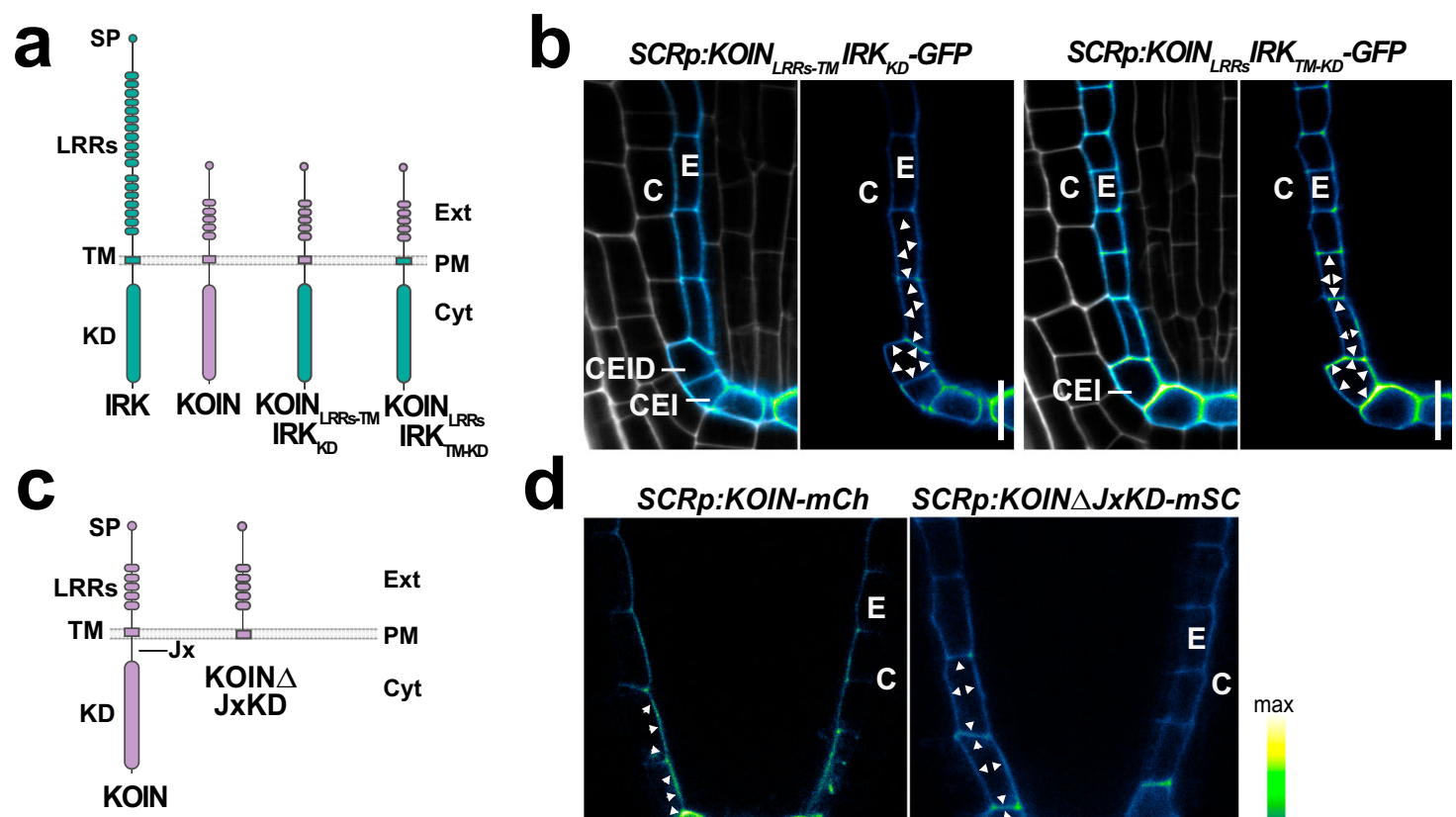

d
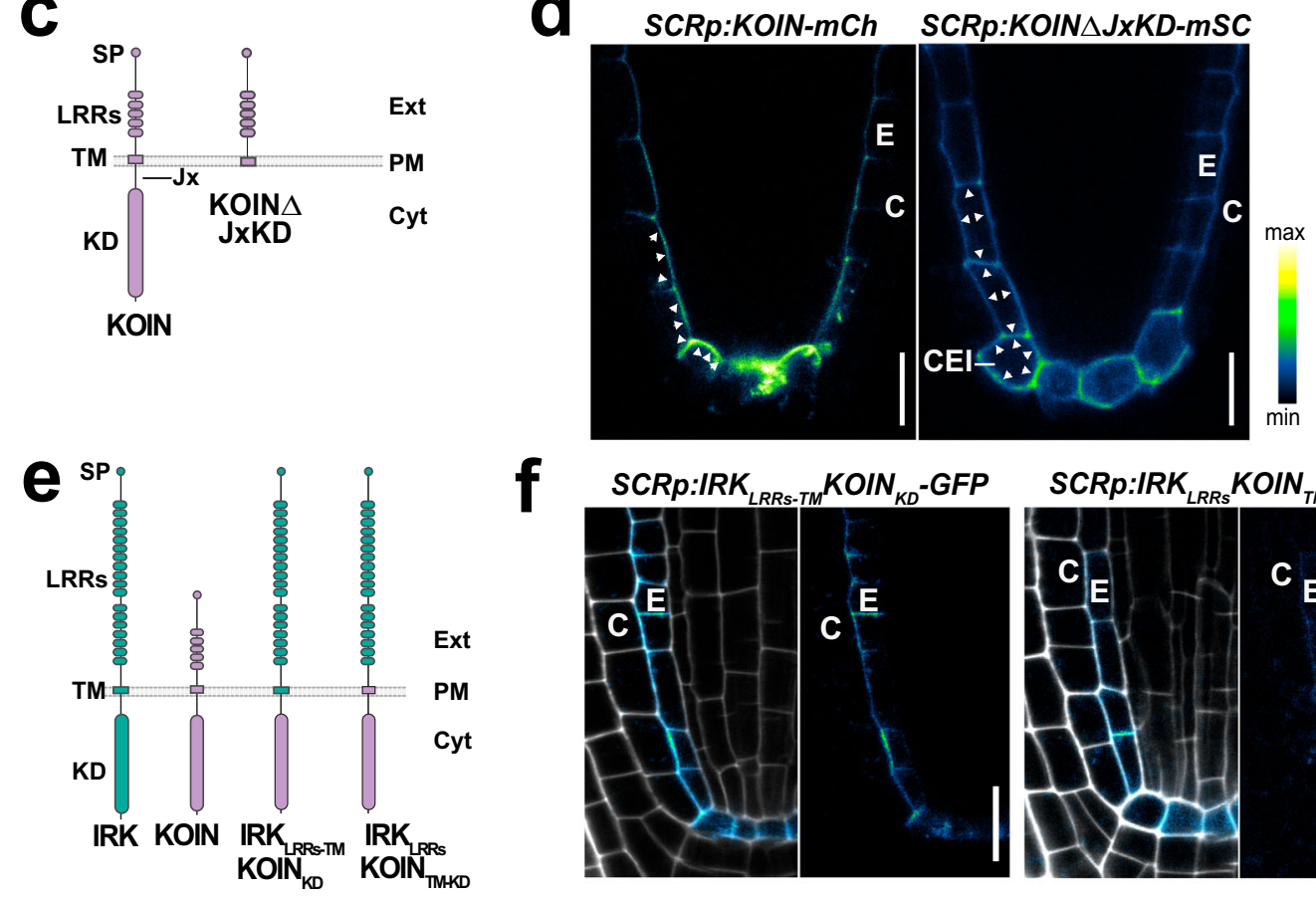

1
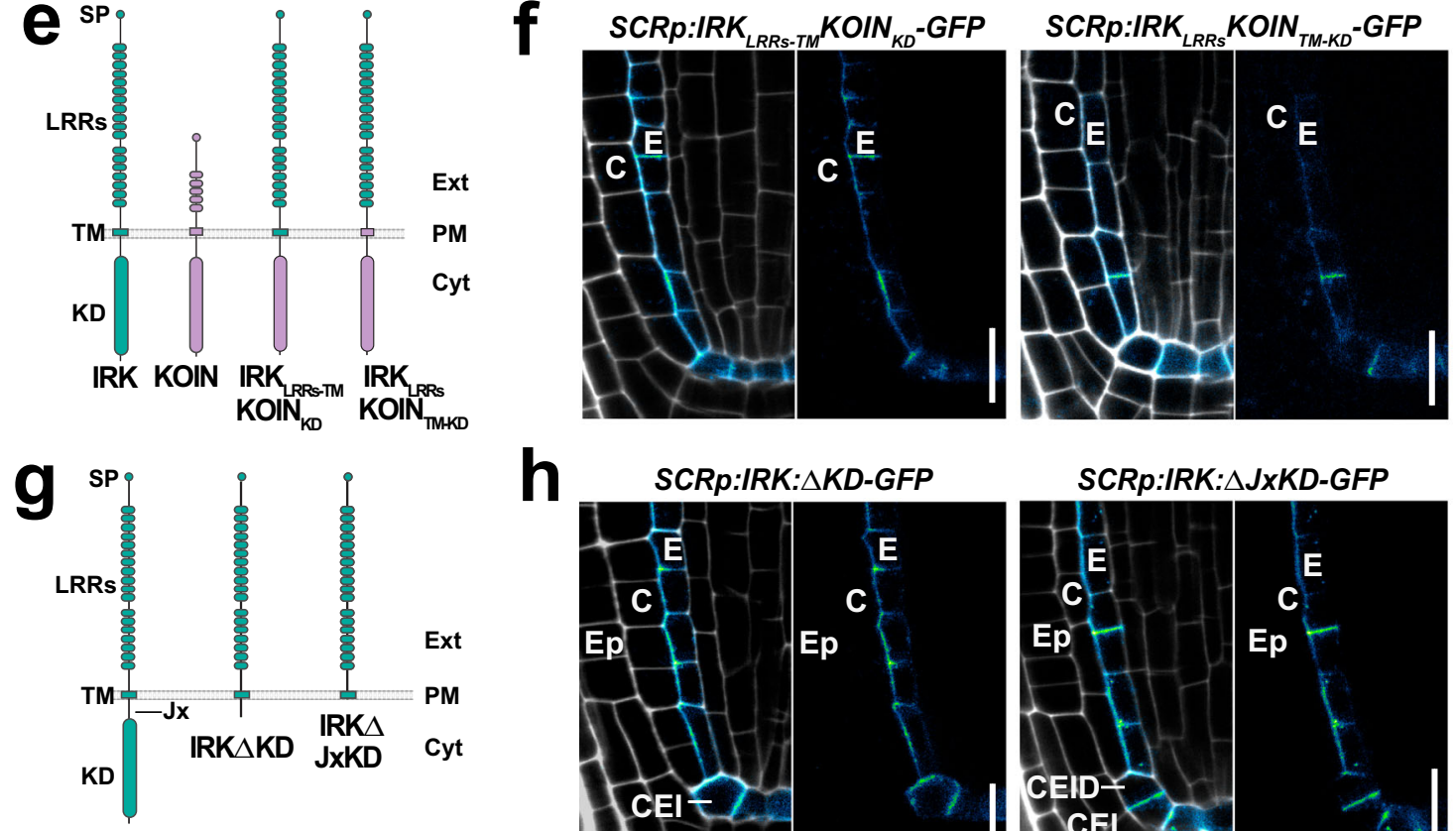

h
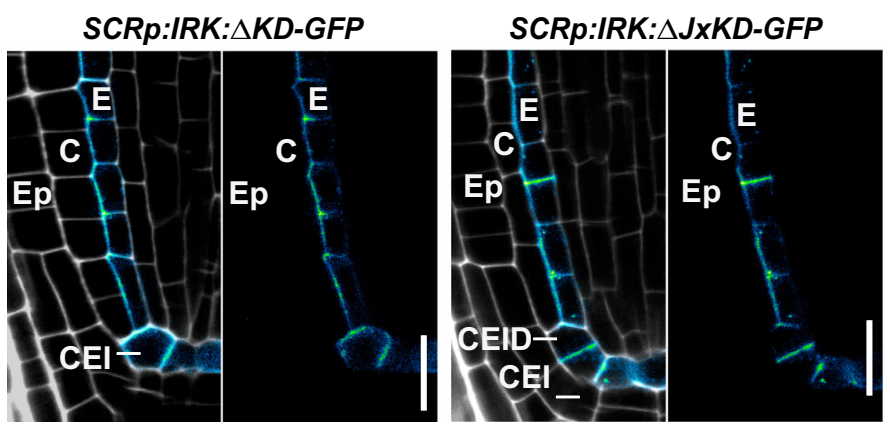

Fig. 4 KOIN and IRK sorting determinants reside in different portions of the protein. a Diagrams of IRK (green) and KOIN (purple) proteins with their extracellular signal peptide (SP) and LRR domains, transmembrane (TM) domain, and kinase domain (KD), and the KOIN-IRK protein chimeras. b Confocal micrographs showing WT root tips expressing SCRp driven KOIN-IRK chimeras with adjacent panels showing GFP + PI merged or GFP alone (GFP intensity color scale, PI gray). Arrowheads indicate the non-polar distribution of this chimera. c Diagrams of KOIN full-length and C-terminal truncation missing the juxtamembrane (Jx) domain and Jx and KD (JxKD). d Confocal images WT root tips expressing SCRp-driven KOIN-mCh (left) and KOIN $\Delta$ JxKD-mSCarlet (mSC, right) with FP shown in intensity color scale. Arrowheads indicate protein distribution. e Diagrams of IRK (green) and KOIN (purple) protein domains and the IRK-KOIN chimeras. $\mathbf{f}$ Confocal images of WT root tips expressing SCRp driven IRK-KOIN chimeras and stained with PI (gray) (merged, left) and GFP fluorescence alone (intensity color scale, right). $\mathbf{g}$ Diagrams of IRK full-length and C-terminal truncations missing the KD or the Jx and KD (JXKD). h Confocal images of WT root tips expressing SCRp driven IRK truncations with adjacent panels showing GFP + PI merged (left) or GFP alone (right). The images are representative of the observations from experiments performed at least three times with at least 15 plants per genotype and at least 3 independent transgenic lines were analyzed. Scale bars: $10 \mu \mathrm{m}$. Abbreviations: (Ep) epidermis; c cortex; e endodermis, (CEI) cortex/endodermal initial, and (CEID) CEI daughter. 
IRK $\Delta K D$ is polarized and partially rescues phenotypes in irk-4. In mammalian cells, homodimerization and clustering of certain single-pass transmembrane receptors are linked to the establishment of lateral polarity ${ }^{42,43}$. In plants, LRR-RLKs are putative ligand-binding receptors and act as co-receptors by homodimerizing or interacting with other proteins through their extracellular, TM, or cytoplasmic domains ${ }^{44,45}$. As IRK $\Delta$ KD-GFP shows the polar distribution in WT, we wanted to eliminate the possibility that homodimerization or clustering with endogenous IRK was the driver of IRK $\Delta$ KD-GFP polarization. In the absence of endogenous IRK (in irk-4) truncated IRK $\Delta$ KD retained its polar localization (Fig. 5g) and, remarkably, endodermal or endogenous expression of IRK $\Delta \mathrm{KD}$-GFP rescued the irk-4 endodermal LAD phenotype. The IRK $\triangle K D$-GFP roots still showed additional periclinal cell divisions and an enlarged stele (Fig. 5c, g-j: Supplementary Fig. 11), which is similar to the phenotypes of irk-1 (Fig. 5f, h-j), a weaker, insertional allele with a premature stop codon that, if translated, would be comparable to IRK $\Delta \mathrm{KD}^{15}$. Furthermore, only expression of IRK-GFP fully rescued the increased endodermal periclinal cell divisions, increased stele area, and LADs observed in irk-4 ${ }^{15}$ (Fig. 5a, b, d, e, $\mathrm{h}-\mathrm{j}$ ). These results indicate that the IRK extracellular domain directs polarization at the PM and in the absence of the $\mathrm{KD}$ retains partial function in the repression of longitudinal anticlinal divisions of endodermal cells.

The KOIN/IRK chimera enhances irk root patterning defects. Our results indicate that the IRK cytoplasmic domain is dispensable for polarization, but necessary to repress the endodermal periclinal divisions and stele enlargement in irk-4. To further assess the functional relevance of IRK-KD in the irk-4 background, we analyzed SCRp-driven endodermal expression of the nonpolar $\mathrm{KOIN}_{\mathrm{LRRsTM}} \mathrm{IRK}_{\mathrm{KD}}$-GFP chimera (Fig. 6; Supplementary Fig. 12f). The dysregulated endodermal cell divisions of irk-4 were not rescued, and additional cellular phenotypes were observed. In the longitudinal axis, cell files with SCRp activity appear discontinuous (Fig. 6c, d). In the radial axis, cells with SCRP activity are adjacent to cells that appear epidermal by position and morphology. Coincidentally, many of those SCRpexpressing cells have also undergone repeated LADs (Fig. 6c, d; Supplementary Fig. 12f). It is possible that excessive endodermal LADs lead to the displacement of the cortex cells resulting in a gap, where endodermal and epidermal cells are immediately adjacent. However, many of these SCRp-expressing cells, based on their position and (pre-division) morphology, appear to have once been cortex cells, suggesting the fate of those cells has been altered. In either case, these results indicate disorganization of root GT, including boundary disruption between cell types. Furthermore, cells with cortex morphology unexpectedly occur interior to SCRp-expressing cells (yellow arrowheads, Fig. 6d). These observations are consistent with general disorganization of longitudinal and radial tissue patterning. Notably, expression of the $\mathrm{KOIN}_{\mathrm{LRRSTM}} \mathrm{IRK}_{\mathrm{KD}}-\mathrm{GFP}$ chimera in WT or koin-1 does not generate additional abnormal phenotypes indicating that this enhanced disorganization occurs specifically in irk-4 (Fig. $4 \mathrm{f}$ and Supplementary Fig. 12d, f).

We were able to exclude that the non-polar KOIN extracellular domain was causal for the phenotypic enhancement of irk-4 (Supplementary Fig. 12a, f). Additionally, expression of fulllength KOIN from $S C R p$ in irk-4 did not result in enhanced phenotypes (Fig. 6e, f), nor did IRK expression in koin-1 (Supplementary Fig. 12c, e, f). Hence, we reasoned that these remarkably enhanced phenotypes can be fully attributed to the expression of $\mathrm{KOIN}_{\mathrm{LRRSTM}} \mathrm{IRK}_{\mathrm{KD}}$ in irk-4. The chimeric nature of the protein suggests that in irk-4 the non-polarly localized KOIN
LRRs perceive signals that are wrongly transduced by a non-polar IRK-KD, leading to disorganized cell layers on either side of the endodermis, which disrupts root radial patterning.

The defects in cell division and tissue patterning generated by the chimera together with the misregulated cell division phenotypes of koin and irk single mutants supports a hypothesis where bi-directional cues converge on the endodermis and are perceived by polarized receptors that modulate specific cell divisions and participate in coordinating cell proliferation and patterning across the entire root meristem.

\section{Discussion}

The establishment and maintenance of polar protein localization at the PM during dynamic cellular events, such as cell division, remain a matter of study. Upon proliferative epidermal cell divisions PIN1, PIN2, and BOR1 are trafficked to the new PMs by non-polar secretion. After cytokinesis, the processes of endocytosis, recycling or degradation, and secretion of newly synthesized proteins, finally, establish shootward/rootward polarity of PIN1/ PIN2 and lateral polarity of BOR1 $1^{4,6,7,46}$. In contrast to these, we find that during proliferative divisions IRK and KOIN have different spatiotemporal accumulation at new PMs indicating distinct targeted secretion mechanisms. A battery of regulatory proteins act at different stages of cell plate formation and are likely involved in spatiotemporal differences in the delivery of specific cargos ${ }^{47-49}$. Additionally, post-cytokinetic polarization of IRK and KOIN depends only on the targeted secretion of de novo protein with their polar delivery occurring via differentially regulated secretion pathways.

During the periclinal formative division of the CEID, IRK secretion is targeted to new PMs while KOIN is not, thus determining the asymmetric accumulation of these two proteins in the daughter endodermal cell. Our observations are consistent with an active role for the mother cell (the CEID here) in the specific sorting and secretion of proteins to new PMs. The resulting polar distribution of these LRR-RLKs in endodermal daughter cells is maintained as they proliferate in the root meristem.

Polar protein secretion is coordinated by small GTPases, the EXOCYST vesicle tethering complex, and pairs of cognate SNAREs on opposing membranes ${ }^{26}$. We find that IRK secretion proceeds through a BFA-sensitive and ES16-insensitive pathway, contrary to KOIN, which is secreted by a BFA-insensitive and ES16-sensitive pathway (Fig. 3n). BFA targets certain ARF-GEFs impacting ARF small GTPase activity and vesicle formation in the Golgi and certain TGN subdomains, which affects the secretion of numerous cargos during and after cell division ${ }^{50-54}$. ES16 targets the small GTPase RABA2A affecting secretion from certain TGN subdomains during interphase and cytokinesis 5,55 . Therefore, KOIN and IRK secretion appear to be sub-compartmentalized into different TGN subdomains. Accordingly, the existence of molecularly, functionally, and spatially distinct TGN subdomains have been experimentally demonstrated ${ }^{56}$. Compartmentalized secretion from the TGN is reported for a handful of polarized proteins ${ }^{5,55,57}$ and contributes to explaining the differential accumulation of IRK and KOIN at the PM during and after proliferative cell divisions.

Trafficking and targeting of TGN-derived vesicles to the appropriate PM domain involve the multiprotein EXOCYST complex ${ }^{58,59}$. Secretion of IRK and KOIN is sensitive to ES2 indicating the involvement of the EXOCYST subunit EXO70A1. This subunit regulates polar exocytosis of a subset of PM proteins indicating selectivity ${ }^{31,32}$. Interestingly, extended treatments with ES2 and Wm, a phosphatidylinositol 3-(PtdIns3K) and 4-(PtdIns4K) kinase inhibitor, decreased IRK and KOIN 
a

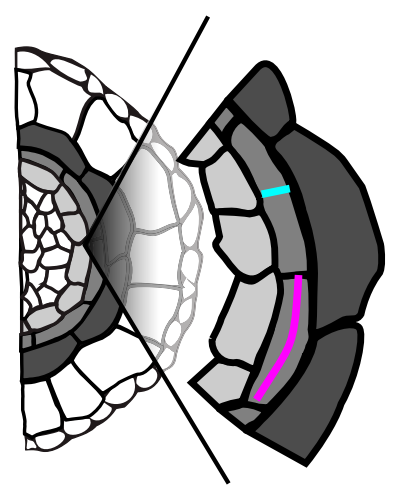

Pericycle Endodermis Cortex
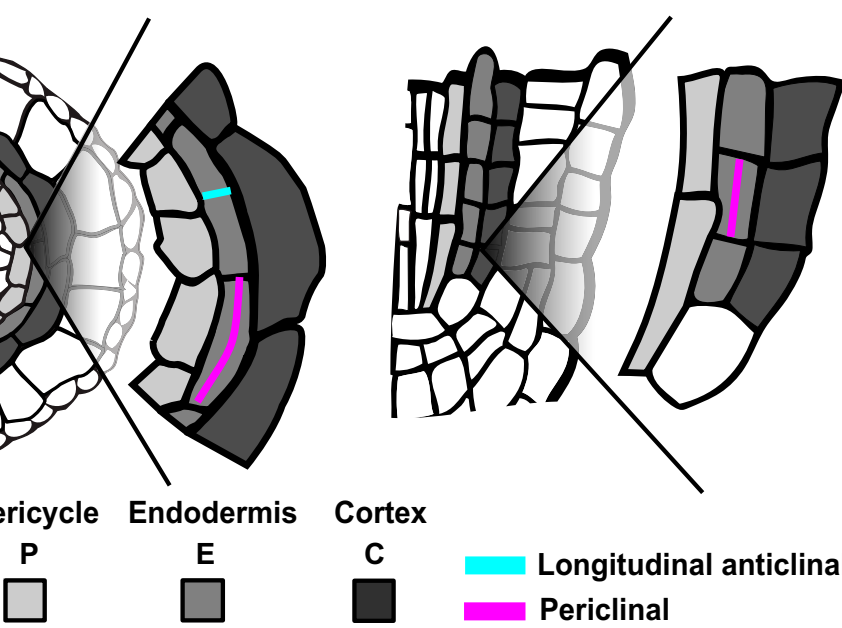

C Longitudinal anticlinal

Periclinal
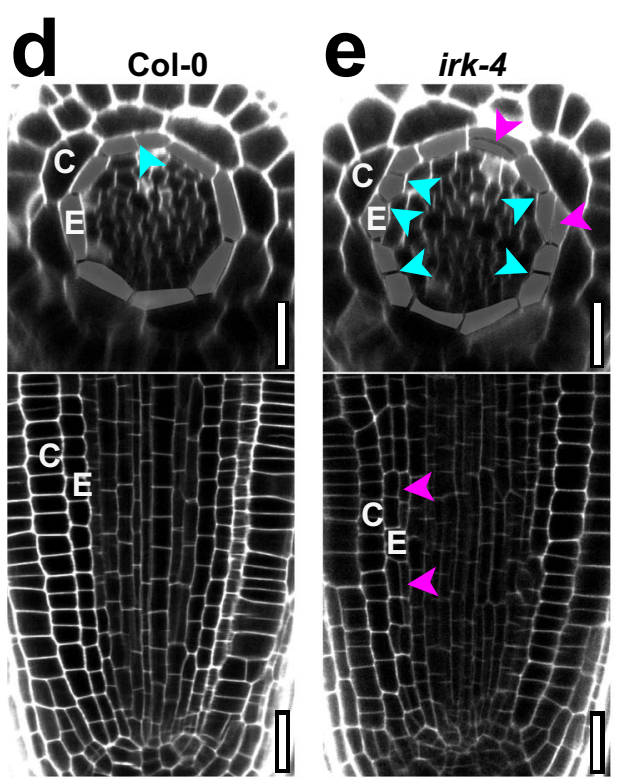

H Endodermal Longitudinal
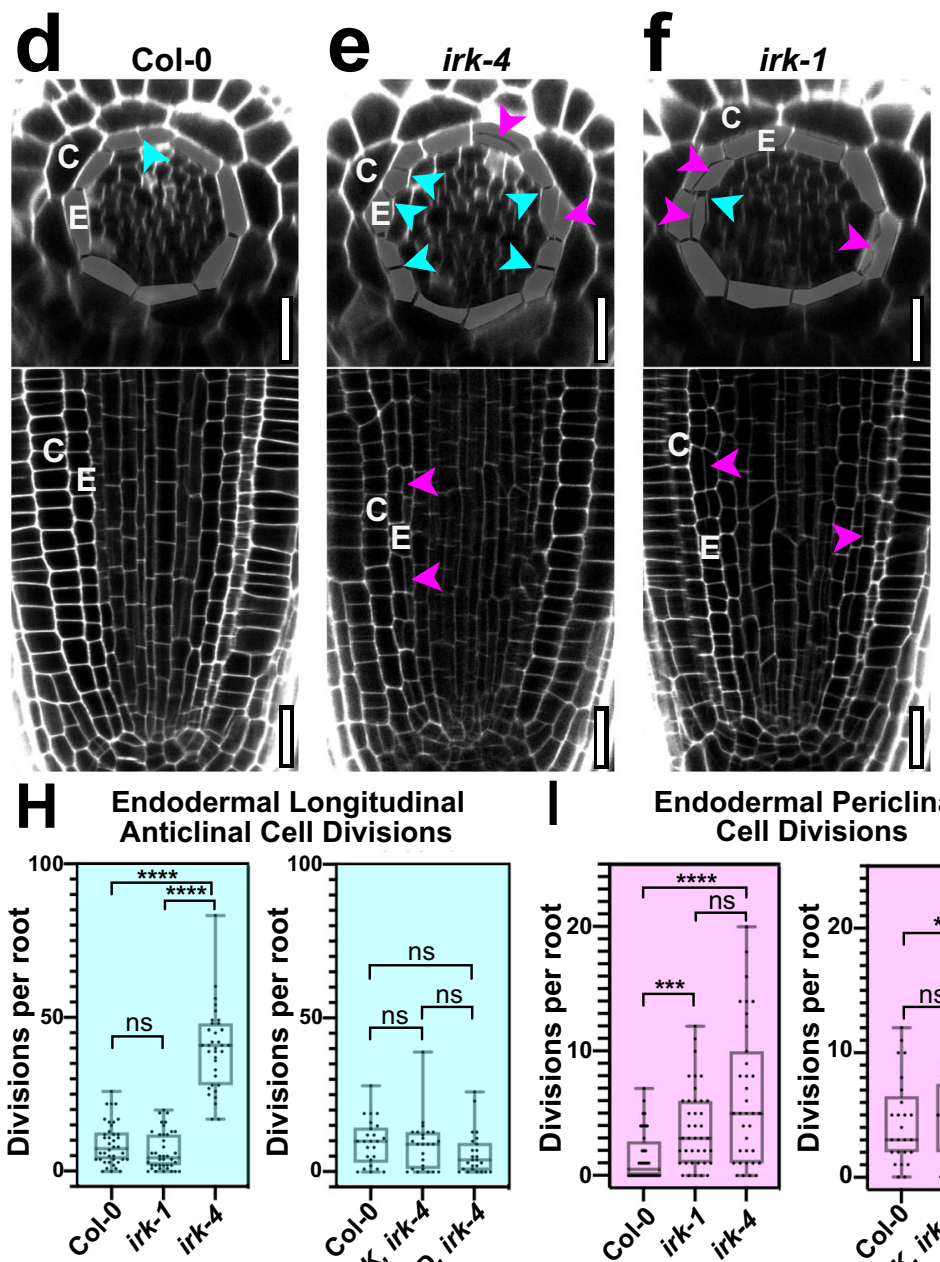

Endodermal Periclinal Cell Divisions

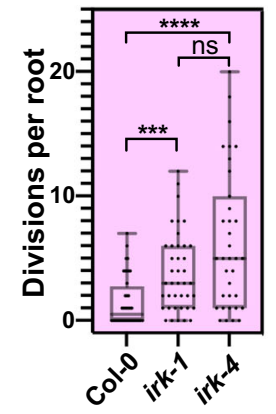

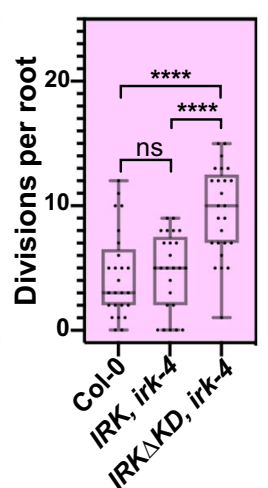
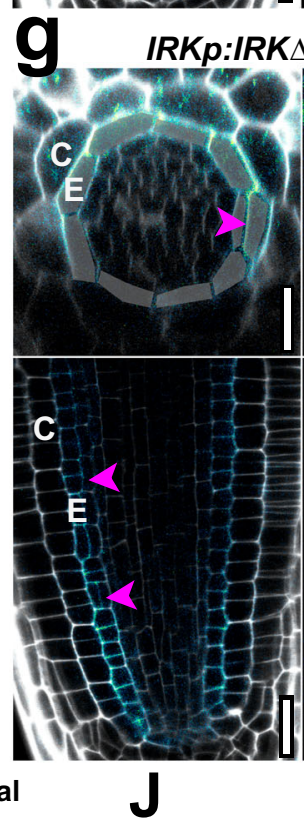

C
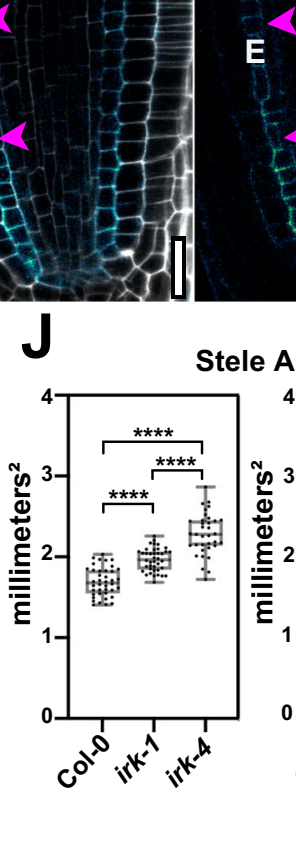

Stele Area

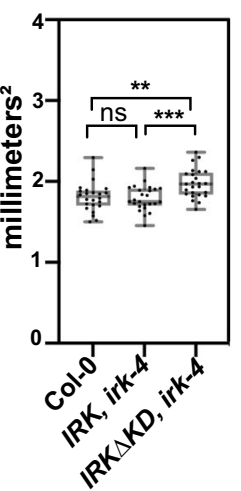

KOIN are secreted through distinct pathways that converge at EXOCYST-mediated targeting.

Secretory proteins contain sorting motifs deciphered by the transport machinery to route them into specific secretion pathways ${ }^{64,65}$. In mammalian systems, sorting signals determining polar secretion are found within the cytoplasmic and TM domains of different single-pass TM proteins ${ }^{66,67}$. Likewise, KOIN sorting 
Fig. 5 IRK $\triangle$ KD is polarized in and partially rescues the cell division phenotypes in irk-4. $\mathbf{a}, \mathbf{b}$ Schematics of portions of the Arabidopsis root tip in $\mathbf{a}$ transverse and $\mathbf{b}$ median longitudinal sections. c-g Confocal images of transverse (upper, with endodermal cells highlighted) and median longitudinal (lower) optical sections from root tips stained with PI (gray) and c, g GFP with intensity color scale and PI (merged) and GFP fluorescence alone. c irk-4 root expressing IRKp:IRK-GFP. d-f Roots of WT, irk-4, and irk-1. $\mathbf{g}$ irk-4 roots expressing IRKp:IRKAKD-GFP. Endodermal longitudinal anticlinal (cyan) and periclinal (magenta) cell divisions are indicated. Quantification of $\mathbf{h}$ longitudinal anticlinal and $\mathbf{i}$ periclinal endodermal cell divisions and (j) stele area of WT, irk-4 and irk-1 roots and irk-4 roots expressing IRK or IRK $\Delta K D$ fusions under IRKp ( $n=20$ roots per genotype). Graphs $\mathbf{h}-\mathbf{j}$ show representative results of experiments performed in $\geq 3$ independent replicates with similar significant results, boxes delimit upper and lower quartiles, median values are indicated with a line, and single values are shown as dots. Bars indicate max/min values and 1-4 stars statistical significance $P$ value $<0.05$ (Brown-Forsythe oneway ANOVA test). Scale bars: $20 \mu \mathrm{m}$.

determinants are present in its cytoplasmic domain. However, none of the canonical cytoplasmic sorting motifs described in plants ${ }^{14,65}$ or other systems ${ }^{68}$ are present within the KOIN cytoplasmic domain indicating that specific, novel motifs direct its polar accumulation. Additionally, although KOIN is described as an inactive kinase $^{69}$, its cytoplasmic domain is essential for function.

IRK sorting determinants, unexpectedly, reside in its extracellular domain. Therefore, a luminal recognition system must exist in the plant endomembrane system to properly direct the polar accumulation of IRK $\triangle \mathrm{KD}$. IRK has 18 LRRs in the extracellular domain that likely participates in protein-protein interactions, therefore luminal associations may occur in the secretory pathway and assist delivery of IRK $\triangle K D$ to the PM. Such is the case for FERONIA, a transmembrane RLK, that interacts with two glycosylphosphatydilinositol-anchored proteins (GPI-AP) at the ER for efficient secretion of the complex to the PM ${ }^{70}$. FERONIA is retained in the ER in the absence of GPI-AP or upon deletion of specific extracellular domains required for GPI-AP interaction, suggesting they act as chaperones facilitating ER exit. Deletions in the IRK extracellular domain also result in ER retention. Further research is necessary to determine if a similar chaperone-driven mechanism facilitates IRK exit from the ER.

The chimeras IRK $\mathrm{LRRs}_{\mathrm{L}-\mathrm{TM}} \mathrm{KOIN}_{\mathrm{KD}}$-GFP and $\mathrm{IRK}_{\mathrm{LRRs}} \mathrm{KOIN}_{\mathrm{TM}}$ $\mathrm{KD}^{-G F P}$ showed IRK-like polarization, demonstrating the hierarchical importance of the sorting signals in the IRK extracellular domain over those found in the KOIN KD. These results reveal fascinating paths for further investigation into the precise mechanisms that recognize these sorting determinants.

Once polarized at the PM, the interaction of IRK $\triangle \mathrm{KD}$ with other proteins or extracellular components likely explains its ability to rescue the endodermal LADs, but not the periclinal cell divisions or enlarged stele phenotypes of irk-4. Strikingly, the irk-1 allele, which is predicted to express an IRK $\Delta \mathrm{KD}$-like protein, exhibits similar phenotypes and, like irk-4, shows altered CYCD6;1p activity. This indicates that, although IRK is described as an inactive kinase ${ }^{69}$, its $\mathrm{KD}$ is functionally important; consequently, only full-length IRK completely rescues irk-4 root phenotypes. These results indicate partitioning of IRK function where putative interactions through the extracellular domain repress endodermal LADs, while those of the IRK cytoplasmic domain regulates periclinal endodermal divisions and stele enlargement.

Endodermal expression of the non-polarly localized $\mathrm{KOIN}_{\text {LRRs- }}$ $\mathrm{TM}_{\mathrm{IRK}} \mathrm{KD}^{-\mathrm{GFP}}$ chimera in irk-4 results in cellular disorganization within and outside the endodermis, suggesting a non-cellautonomous influence on cell division and perhaps cell identity. This chimera may inappropriately perceive signals due to its nonpolar localization and/or elicit an incorrect downstream response impacting root radial patterning. However, further research is necessary to fully establish this hypothesis. As IRK and KOIN are implicated in cell division control at the endodermis, it is tempting to propose that downstream of the SHR transcriptional network these two LRR-RLKs, polarized at opposites sides of the endodermal PM, interpret bi-directional information that modulates root cell proliferation in the radial and longitudinal axes. Our observations are consistent with others that indicate the endodermis non-cell autonomously regulates developmental events in the root meristem ${ }^{71,72}$. Identification of the extra- and intracellular interactors of IRK and KOIN will be critical to further untangle the precise developmental roles of these polarized LRR-RLKs.

\section{Methods}

Plant material and growth conditions. Arabidopsis thaliana ecotype Col-0 was used as wild type (WT). The koin-1 (WiscDsLox_439H07) and koin-2 (GabiKat822B12) alleles were obtained from the ABRC (Arabidopsis Resource Center). The genotypes IRKp:IRK:GFP, SCRp:IRK-GFP, CO2p:IRK-GFP, pub4-1 SCRp:IRK:GFP, irk-4, irk-1, SCRp:IRK:GFP irk-4, IRKp:IRK:GFP irk-4, pSCR:erGFP, scr-4 were previously described ${ }^{15}$. Unless otherwise specified, seeds were surface sterilized by chlorine gas, sown on solid Arabidopsis medium 1X Murashige and Skoog basal salts (Caisson labs), $0.5 \mathrm{~g} / \mathrm{L} \mathrm{MES,} 1 \%$ sucrose, and $1 \%$ agar (Difco), at $\mathrm{pH} 5.7$; then stratified at $4{ }^{\circ} \mathrm{C}$ for at least 2 days prior to transfer to a $16 / 8 \mathrm{~h}$ illumination regime in a Percival growth chamber at a continuous temperature of $22^{\circ} \mathrm{C}$. Seedlings were grown on vertically oriented plates sealed with parafilm or micropore tape for 4-7 days prior to analysis.

Plasmid construction. Expression vectors (Supplementary Table 2) were constructed utilizing Invitrogen Multisite Gateway ${ }^{\oplus}$ technology (Carlsbad, USA). Briefly pENTR vectors containing the various promoters, coding regions, and fluorescent tags were recombined into the destination vectors to generate plant expression vectors for transformation.

mSCARLETi (in pUC57) was received from Dr. David Nelson (University of California, Riverside). The mSCARLET coding sequence (754 bp) was amplified with attB2/attB3 recombination sites added in the primers (Supplementary Table 3); then, recombined via BP clonase (Invitrogen, Cat \#11789-013) into the pDONR ${ }^{\mathrm{TM}} \mathrm{P} 2 \mathrm{r}-\mathrm{P} 3$ vector.

KOIN is encoded by At5g58300 and all components were cloned by PCR from WT genomic DNA and verified by sequencing. The KOIN promoter (1709 bp) fragment containing the entire intergenic region between the stop codon of At5g58290 and the KOIN start codon was amplified (Supplementary Table 3) and recombined into the pENTR ${ }^{\mathrm{nt}}$ DIRECTIONAL TOPO ${ }^{\oplus}$ (pENTR-D-TOPO) vector. The 2048 bp KOIN genomic region, including intron 2 ( $86 \mathrm{bp}$ ) and the coding region, was amplified (Supplementary Table 3 ) and recombined into the pENTRD-TOPO vector.

To generate IRK extracellular domain deletions the pENTR-D-TOPO vector containing the IRK genomic sequence was digested with restriction enzymes to remove specific regions. Digested vectors were purified, re-ligated using Promega T4-DNA ligase, and the reading frame was verified by DNA sequencing. Specifically, IRK $\triangle$ LRR1 was generated by digestion with XbaI, IRK $\Delta$ LRR17,18 by digestion with SalI and SmaI, and IRK $\Delta$ LRR1-18 by digestion with BglII and SmaI. In the case of IRK $\Delta$ LRR17,18 and IRK $\Delta$ LRR1-IRK $\Delta 18$, DNA polymerase I, large (Klenow) fragment (New England Biolabs) was used to fill in the overhangs prior to ligation. The KOIN extracellular domain deletion was similarly generated by digestion and ligation. To generate the SCRp:KOIN $\Delta$ LRR:GFP expression vector, the expression vector containing full-length KOIN-GFP driven by SCRp (SCRp:KOIN:GFP) was digested with EcoRI and AccIII (BspEI), purified, then overhangs were filled in with Klenow, and ligated together. Deletions of IRK cytoplasmic domains (IRK $\triangle \mathrm{KD}$ and IRK $\Delta \mathrm{JxKD}$ ) were cloned by PCR from WT genomic DNA using primers designed to amplify from the IRK start codon up to the beginning of the $\mathrm{KD}(\Delta \mathrm{KD}, 2115 \mathrm{bp}, 674 \mathrm{aa})$ or just through the transmembrane domain $(\triangle \mathrm{JxKD}, 2007 \mathrm{bp}, 638 \mathrm{aa})$ and the fragments were recombined into the pENTR-D-TOPO vector. The cytoplasmic KOIN deletion KOIN $\triangle \mathrm{JxKD}$ was similarly generated by amplifying the region from its start codon through the transmembrane domain $(\Delta \mathrm{JxKD}, 942 \mathrm{bp}, 314 \mathrm{aa})$.

To create the different chimeric proteins, DNA fragments of interest were cloned by PCR from cDNA clones (IRK: N3G56370ZEF, KOIN: N5G58300ZEF, and ER: N2G26330ZEF (ABRC). Restriction enzyme sites were designed into the primers to allow the specific coding portions from each gene to be ligated in-frame. The PCR-amplified fragments were gel purified and then digested with their 

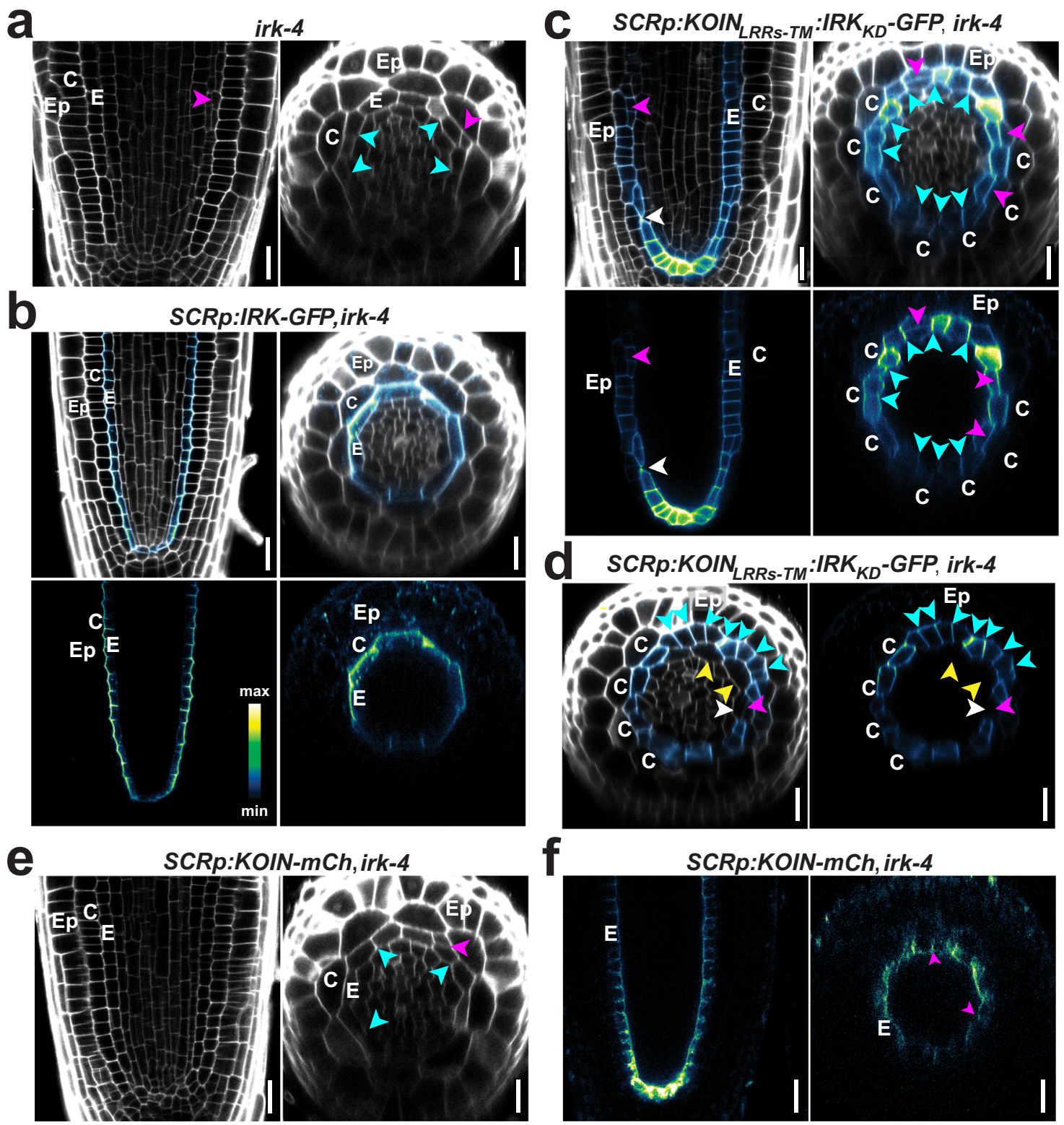

Fig. 6 Endodermal expression of KOIN-IRK chimera results in additive cell division phenotypes in irk-4. a-f Confocal images of median longitudinal and/or transverse sections of roots stained with $\mathrm{PI}$ (gray) and/or fluorescence (intensity color scale). a irk-4 roots showing endodermal longitudinal anticlinal (cyan arrowheads) and periclinal (magenta arrowheads) cell divisions. b irk-4 roots expressing SCRp:IRK:GFP (GFP + PI merged) and GFP alone. c, $\mathbf{d}$ irk-4 roots expressing SCRp:KOIN LRRS $_{\text {IRK }} K_{T M-K D}-$ GFP at $\mathbf{c} 7$ and $\mathbf{d} 4$ d.p.s. showing GT disorganization. SCRp driven GFP signal is detected in cells that positionally correspond to the cortex as they are immediately adjacent to the epidermis but have undergone many longitudinal anticlinal cell divisions. White arrowheads indicate an apparent discontinuity in endodermal cell files/rings. d A root with cells immediately interior to the epidermis that express the SCRp driven chimera with cells interior to those that morphologically resemble cortex (yellow arrowheads). e, $\mathbf{f}$ irk-4 roots expressing SCRp:KOIN-mCh $\mathbf{g}$ stained with PI (gray) and showing h KOIN-mCh localization ( $\mathrm{mCh}$ intensity false-colored). Images correspond to representative observations of experiments replicated at least 3 times using $\geq 15$ plants/genotype. Scale bar: $20 \mu \mathrm{m}$.

respective enzyme (see Supplementary Table 3). The digested fragments were gelpurified then ligated with T4 DNA Ligase and the resulting product was PCR amplified. The products from the final PCR amplification were recombined into the pENTR-D-TOPO vector and verified by sequencing. For example, to generate the KOIN LRRs-TM:IRK $_{\mathrm{KD}}$ chimera, the KOIN fragment was PCR amplified from its ATG through the transmembrane domain with the reverse primer containing an EcoRV cut site such that the 3 ' end of this KOIN fragment contains a coding region for its extracellular and transmembrane domain, plus a few amino acids that precede the KD. Complementary to this, the forward primer to amplify the IRK $\mathrm{KD}$ contains an EcoRV cut site. After amplification and digestion of each with EcoRV, the fragments can be ligated with T4 DNA ligase, thus creating the inframe $\mathrm{KOIN}_{\mathrm{LRRS}-\mathrm{TM}}: \mathrm{IRK}_{\mathrm{KD}}$ chimera.
Generation of transgenic plants. Validated expression vectors were transformed into Agrobacterium tumefaciens strain GV3101 for transformation into Arabidopsis plants by the floral dip method (Clough and Bent, 1998). Transformed plants with resistance to Basta (dpGreen-BarT) or Kanamycin (dpGreen-KanT) were selected using standard methods. T2 lines exhibiting a resistant-to-sensitive ratio of 3:1, indicating a single insertion locus, were selected for propagation. T3 progeny with $100 \%$ resistant seedlings (homozygous) were selected for analysis. At least three independent lines for each reporter showing comparable fluorescence levels and protein distribution profiles were used for subsequent analysis.

Homozygous plants expressing the different constructs were crossed by standard methods. Two independent, representative pCO2:KOIN:GFP lines in Col0 were crossed with $s c r-4$ heterozygous plants. In the F2 generation, scr-4 mutant 
seedlings were identified by their root phenotype and checked by confocal microscopy for KOIN:GFP. Independent lines expressing SCRp:KOIN-mCh and pSCR:IRK:GFP (each in Col-0) were also reciprocally crossed. Among the F4 progeny, those with $100 \%$ Basta and Kanamycin resistant seedlings showing IRK and KOIN fluorescence at comparable levels were selected for further analyses.

Chemical treatments. Seedlings were grown on $1 \times$ MS solid medium plates sealed with tape for 6 d.p.s. For treatments of up to $6 \mathrm{~h}$, seedlings were transferred to 24 well plates (5-8 seedlings per well) containing $1 \mathrm{~mL}$ of $1 \times$ MS liquid medium containing $50 \mu \mathrm{M}$ BFA, $40 \mu \mathrm{M}$ ES2, $30 \mu \mathrm{M}$ Wm, $50 \mu \mathrm{M}$ CHX, $20 \mu \mathrm{M}$ LatB, $20 \mu \mathrm{M}$ Oryzalin, combinations of these chemicals, or the solvent control. For $24 \mathrm{~h}$ BFA treatments, seedlings were transferred to $1 \times$ MS solid medium to avoid precipitation of the chemicals. BFA was added to $1 \times$ MS agar medium (cooled to $55^{\circ} \mathrm{C}$ ) at a final concentration of $50 \mu \mathrm{M}$ before pouring the plates. Control plants were incubated in media containing equal volumes of solvent. Stock solutions of $40 \mathrm{mM}$ BFA (Sigma-Aldrich), $10 \mathrm{mM} \mathrm{ES16}{ }^{5}, 40$ mM ES2 ${ }^{31}, 33 \mathrm{mM}$ Wm (SigmaAldrich), $50 \mathrm{mM}$ CHX (Sigma-Aldrich), $20 \mathrm{mM}$ LatB (Sigma-Aldrich) and $50 \mathrm{mM}$ Oryzalin (Sigma-Aldrich) were prepared using DMSO as a solvent. The imaging was carried out in the presence of the liquid media, plus the respective chemicals/ solvents. At least 20 plants per condition were analyzed. Each treatment was replicated at least three times to assure reproducibility.

Confocal microscopy and image analysis. Roots were stained with $\sim 10 \mu \mathrm{M}$ PI solubilized in water for 1-2 min before visualization. Roots were stained with $10 \mu \mathrm{M}$ FM4-64 diluted in liquid MS media for 5-10 min, to allow sufficient dye penetration to visualize the PM of endodermal cells.

Imaging was performed on a Leica SP8 upright confocal microscope equipped with a water-corrected $40 \times(1.1$ numerical aperture (NA)) objective. Images were generated using PMT and HYD detectors with the pinholes adjusted to 1 Airy unit for each wavelength and system settings as follows: GFP excitation $488 \mathrm{~nm}$, emission at $492-530 \mathrm{~nm}$; YFP excitation $514 \mathrm{~nm}$, emission at $515-550 \mathrm{~nm}$; mCh or $\mathrm{mSC}$ excitation $594 \mathrm{~nm}$, emission at 585-660 nm, PI excitation $536 \mathrm{~nm}$, emission at 585-660 nm, and FM4-64 excitation $488 \mathrm{~nm}$, emission at 600-660 nm. In-line sequential scanning was performed when GFP and $\mathrm{mCh} / \mathrm{mSC}$ or FM4-64 fluorescence was collected in the same root to avoid signal bleed through. Image processing was performed using the LAS X software or ImageJ software (imagej.nih.gov/ij/). For FRAP experiments, image acquisition was performed in the Zeiss LSM 880 inverted microscope using a $40 \times(1.1$ NA) water immersion objective and the ZEN (black edition) software to set the parameters and perform the quantifications.

The two-dimensional graphs displaying IRK-GFP and KOIN-mCh pixel intensities along newly formed PMs during cell divisions were generated with the plot profile plugin in ImageJ. The X-axis represents the distance of the line drawn at the membrane and the $y$-axis is the pixel intensity along that line. To compare IRK-GFP or KOIN-GFP distribution with that of FM4-64 at the PM of CEI and CEID, we selected a rectangular area and used the surface plot plugin in ImageJ to produce a three-dimensional graph of pixel intensities. Additionally, the signal intensity of IRK-GFP and KOIN-mCh after ES2 or Wm treatments was quantified in ImageJ using the Measure tool and applying the formula: corrected signal intensity $=$ integrated density - (area selected $\times$ mean fluorescence of background readings). Fluorescence intensity detected in the root meristem just above the QC from control images (control values) was used as a reference to provide a percentage of signal intensity when compared to the same area in the respective treatments.

For each quantification, the raw data was exported, and the graphic output was produced using Prism (Graphpad, San Diego, CA).

Phenotypic analyses. Seedlings of the various genotypes being compared were grown side-by-side on $0.2 \times$ MS agar plates sealed with parafilm. At 5, 6, and/or 7 d.p.s., roots were stained with $\sim 10 \mu \mathrm{M}$ PI solubilized in water for 1-2 min and visualized via laser scanning confocal microscopy (Leica Sp8). Z-stacks of the root meristem of 10-15 seedlings per genotype were acquired for 2-3 biological replicates. Endodermal LADs were observed by analyzing transverse optical sections with the orthogonal sectioning tool of the LAS X software. The number of endodermal LADs per transverse section from the QC up to 15 cells above the QC were counted. Therefore, the total number of LADs is the sum of these divisions within this region. Periclinal divisions in the endodermis were counted within the range of $\mathrm{QC}+120 \mu \mathrm{m}$ by examining longitudinal and transverse optical sections. Stele area was quantified in ImageJ by tracing a polygon along the inner surface of endodermal cells at $120 \mu \mathrm{m}$ above the QC and then the polygon area was calculated by the software.

To measure root length, all plates were scanned, and the resulting images were analyzed using Image J software. To measure root meristem size, the number of cortical cells from the QC to the first elongated cell was counted. For each experiment, at least 15 roots were analyzed, and the mean and standard deviation were calculated. The data were exported and analyzed using Excel and the graphic output was produced using Prism (Graphpad).
Cell division quantification. Seedlings of the various genotypes being compared were grown side-by-side on $0.2 \times$ MS agar plates sealed with parafilm for 7 d.p.s. Seedlings were fixed using $4 \%$ paraformaldehyde in MTSB ( $50 \mathrm{mM}$ PIPES, $5 \mathrm{mM}$ EGTA, $5 \mathrm{mM} \mathrm{MgSO} 4, \mathrm{pH}$ 7) for $1 \mathrm{~h}$ and washed three times in MTBS. Cells were permeabilized by treatment for $1 \mathrm{~h}$ in $10 \%$ DMSO, 3\% IGEPAL (Sigma) in MTSB ( $\mathrm{pH} 7$ ). Roots were then stained with $\sim 10 \mu \mathrm{M}$ PI solubilized in water for $5 \mathrm{~min}$ and visualized via laser scanning confocal microscopy (Leica Sp8). Z-stacks of the root meristem of 10-15 seedlings per genotype were acquired for three biological replicates. From the QC up to $250 \mu \mathrm{m}$ cortex and endodermal cells in metaphase, anaphase or telophase were scored. The data were exported and analyzed using Excel and the graphic output was produced using Prism (Graphpad).

RT-qPCR analysis. Total RNA was isolated from 20 whole seedlings per genotype in three independent biological replicates for each koin allele and the corresponding WT controls using Qiagen's RNeasy Plant Mini Kit after 7 d.p.s. on $1 \times$ MS agar medium sealed with parafilm. First-strand cDNA was synthesized using RevertAid First Strand cDNA Synthesis Kit and the Oligo(dT)18 primer (Thermo Scientific). qRT-PCR reactions were set up using IQ SYBR Green Supermix (BioRad) and analysis was performed on the CFX Connect Real-Time System. For $K O I N$, three mRNA splicing forms have been described (TAIR10) and, therefore, we used a pair of primers to specifically detect KOIN.1 and another pair to detect KOIN.2 and KOIN.3 (Supplementary Fig. 1A and Supplementary Table 2). Standard curves were performed at least in duplicate. Primer pair efficiency values were calculated for each replicate of the standard curves and the average efficiency was used for subsequent analysis. For each genotype and biological replicate, three technical replicates were included. The expression shown is relative to Col-0 and KOIN transcript levels were normalized to PP2A (Atlg13320) ${ }^{73}$ using the Bio-Rad CFX Manager software 3.1 .

Reporting summary. Further information on research design is available in the Nature Research Reporting Summary linked to this article.

\section{Data availability}

All data generated or analyzed during this study are included in this published article (and its supplementary information files). Source data are provided with this paper. Additional information and materials generated for and/or reported in this article are available from the corresponding author upon request. Source data are provided with this paper.

Received: 25 March 2021; Accepted: 22 December 2021; Published online: 11 January 2022

\section{References}

1. Muroyama, A. \& Bergmann, D. Plant cell polarity: creating diversity from inside the box. Annu. Rev. Cell Dev. Biol. 35, 309-336 (2019).

2. Luschnig, C. \& Vert, G. The dynamics of plant plasma membrane proteins: PINs and beyond. Development 141, 2924-2938 (2014).

3. Langowski, L. et al. Cellular mechanisms for cargo delivery and polarity maintenance at different polar domains in plant cells. Cell Discov. 2, 1-18 (2016). 2.12016

4. Kleine-Vehn, J. et al. Cellular and molecular requirements for polar PIN targeting and transcytosis in plants. Mol. Plant 1, 1056-1066 (2008).

5. Li, R. et al. Different endomembrane trafficking pathways establish apical and basal polarities. Plant Cell 29, 90-108 (2017).

6. Glanc, M., Fendrych, M. \& Friml, J. Mechanistic framework for cell-intrinsic re-establishment of PIN2 polarity after cell division. Nat. Plants 4, 1082-1088 (2018).

7. Men, S. et al. Sterol-dependent endocytosis mediates post-cytokinetic acquisition of PIN2 auxin efflux carrier polarity. Nat. Cell Biol. 10, 237 (2008)

8. Raggi, S., Demes, E., Liu, S., Verger, S. \& Robert, S. Polar expedition: mechanisms for protein polar localization. Curr. Opin. Plant Biol. 53, 134-140 (2020).

9. Takano, J. et al. Polar localization and degradation of Arabidopsis boron transporters through distinct trafficking pathways. Proc. Natl Acad. Sci. 107, 5220-5225 (2010).

10. Alassimone, J., Naseer, S. \& Geldner, N. A developmental framework for endodermal differentiation and polarity. Proc. Natl Acad. Sci. USA 107, 5214-5219 (2010)

11. Zelazny, E. \& Vert, G. Plant nutrition: root transporters on the move. Plant Physiol. 166, 500-508 (2014).

12. Takano, J., Miwa, K., Yuan, L., von Wirén, N. \& Fujiwara, T. Endocytosis and degradation of BOR1, a boron transporter of Arabidopsis thaliana, regulated by boron availability. Proc. Natl Acad. Sci. USA 102, 12276-12281 (2005). 
13. Wang, S. et al. Polar localization of the NIP5;1 boric acid channel is maintained by endocytosis and facilitates boron transport in arabidopsis roots. Plant Cell 29, 824-842 (2017).

14. Yoshinari, A., Kasai, K., Fujiwara, T., Naito, S. \& Takano, J. Polar localization and endocytic degradation of a boron transporter, BOR1, is dependent on specific tyrosine residues. Plant Signal Behav. 7, 46-49 (2012).

15. Campos, R., Goff, J., Rodriguez-Furlan, C. \& Van Norman, J. M. The Arabidopsis receptor kinase IRK is polarized and represses specific cell divisions in roots. Dev. Cell 52, 183-195.e4 (2020).

16. Nakajima, K., Sena, G., Nawy, T. \& Benfey, P. N. Intercellular movement of the putative transcription factor SHR in root patterning. Nature 413, 307-311 (2001).

17. Heidstra, R., Welch, D. \& Scheres, B. Mosaic analyses using marked activation and deletion clones dissect Arabidopsis SCARECROW action in asymmetric cell division. Genes Dev. 18, 1964-1969 (2004).

18. Yu, Q. et al. Cell-fate specification in Arabidopsis roots requires coordinative action of lineage instruction and positional reprogramming. Plant Physiol. 175, 816-827 (2017).

19. Cui, H. et al. An evolutionarily conserved mechanism delimiting SHR movement defines a single layer of endodermis in plants. Science 316, 421-425 (2007).

20. Sozzani, R. et al. Spatiotemporal regulation of cell-cycle genes by SHORTROOT links patterning and growth. Nature 466, 128-132 (2010).

21. Brady, S. M. et al. A high-resolution root spatiotemporal map reveals dominant expression patterns. Science 318, 801-806 (2007).

22. Li, S., Yamada, M., Han, X., Ohler, U. \& Benfey, P. N. High-resolution expression map of the arabidopsis root reveals alternative splicing and lincRNA regulation. Dev. Cell 39, 508-522 (2016).

23. Van Norman JM Asymmetry and cell polarity in root development. Dev. Biol. 419, 165-174 (2016).

24. Kinoshita, A. et al. A plant U-box protein, PUB4, regulates asymmetric cell division and cell proliferation in the root meristem. Development. 142, 444-453 (2015).

25. van Oostende-Triplet, C. et al. Vesicle dynamics during plant cell cytokinesis reveals distinct developmental phases. Plant Physiol. 174, 1544-1558 (2017).

26. Rodriguez-Furlan, C., Minina, E. A. \& Hicks, G. R. Remove, recycle, degraderegulating plasma membrane protein accumulation. Plant Cell 31, 2833-2854 (2019). 2019.

27. Jásik, J. \& Schmelzer, E. Internalized and newly synthesized arabidopsis PINFORMED2 pass through brefeldin A compartments: a new insight into intracellular dynamics of the protein by using the photoconvertible fluorescence protein Dendra2 as a tag. Mol. Plant 7, 1578-1581 (2014).

28. Mao, H., Nakamura, M., Viotti, C. \& Grebe, M. A framework for lateral membrane trafficking and polar tethering of the PEN3 ATP-binding cassette transporter. Plant Physiol. 172, 2245-2260 (2016).

29. Geldner, N. et al. The arabidopsis GNOM ARF-GEF mediates endosomal recycling, auxin transport, and auxin-dependent plant growth. Cell $\mathbf{1 1 2}$ 219-230 (2003).

30. Mossessova, E., Corpina, R. A. \& Goldberg, J. Crystal structure of ARF1• Sec7 complexed with Brefeldin A and its implications for the guanine nucleotide exchange mechanism. Mol. Cell 12, 1403-1411 (2003).

31. Zhang, C. et al. Endosidin 2 targets conserved exocyst complex subunit EXO70 to inhibit exocytosis. Proc. Natl Acad. Sci. USA 113, E41 LP-E41 50 (2016).

32. Lešková, A. et al. Endosidin 2 accelerates PIN2 endocytosis and disturbs intracellular trafficking of PIN2, PIN3, and PIN4 but not of SYT1. PLoS ONE 15, e0237448 (2020).

33. Chalmers, A. D., Whitley, P., Ang, S. F. \& Fölsch, H. The role of secretory and endocytic pathways in the maintenance of cell polarity. Essays Biochem. 53, 29-39 (2012).

34. Bonifacino, J. S. Adaptor proteins involved in polarized sorting. J. Cell Biol. 204, 7-17 (2014).

35. Fölsch, H., Pypaert, M., Maday, S., Pelletier, L. \& Mellman, I. The AP-1A and AP-1B clathrin adaptor complexes define biochemically and functionally distinct membrane domains. J. Cell Biol. 163, 351-362 (2003).

36. Yoshinari, A. et al. Polar localization of the borate exporter BOR1 requires AP2-dependent endocytosis. Plant Physiol. 179, 1569-1580 (2019).

37. Taylor, S. S. \& Kornev, A. P. Protein kinases: evolution of dynamic regulatory proteins. Trends Biochem. Sci. 36, 65-77 (2011).

38. Cowan-Jacob, S. W. Structural biology of protein tyrosine kinases. Cell Mol. Life Sci. 63, 2608-2625 (2006).

39. Hanks, S. K. \& Hunter, T. The eukaryotic protein kinase superfamily: kinase (catalytic) domain structure and classification 1. FASEB J. 9, 576-596 (1995).

40. Castells, E. \& Casacuberta, J. M. Signalling through kinase-defective domains: the prevalence of atypical receptor-like kinases in plants. J. Exp. Bot. 58, 3503-3511 (2007).

41. Meng, X. et al. Differential function of arabidopsis SERK family receptor-like kinases in stomatal patterning. Curr. Biol. 25, 2361-2372 (2015).
42. Clayton, A. H. A., Tavarnesi, M. L. \& Johns, T. G. Unligated epidermal growth factor receptor forms higher order oligomers within microclusters on A431 cells that are sensitive to tyrosine kinase inhibitor binding. Biochemistry 46, 4589-4597 (2007).

43. Nagy, P., Claus, J., Jovin, T. M. \& Arndt-Jovin, D. J. Distribution of resting and ligand-bound ErbB1 and ErbB2 receptor tyrosine kinases in living cells using number and brightness analysis. Proc. Natl Acad. Sci. USA 107, 16524-16529 (2010).

44. Xi, L., Wu, X. N., Gilbert, M. \& Schulze, W. X. Classification and interactions of LRR receptors and co-receptors within the arabidopsis plasma membranean overview. Front. Plant Sci. 10, 1-8 (2019).

45. Zheng, B. et al. EMS1 and BRI1 control separate biological processes via extracellular domain diversity and intracellular domain conservation. Nat. Commun. 10, 1-10 (2019).

46. Yoshinari, A. et al. DRP1-dependent endocytosis is essential for polar localization and boron-induced degradation of the borate transporter BOR1 in Arabidopsis thaliana. Plant Cell Physiol. 57, 1985-2000 (2016).

47. Davis, D. J. et al. The RAB GTPase RABAle localizes to the cell plate and shows distinct subcellular behavior from RABA2a under Endosidin 7 treatment. Plant Signal Behav. 11, e984520 (2016).

48. Park, E. et al. Endosidin 7 specifically arrests late cytokinesis and inhibits callose biosynthesis, revealing distinct trafficking events during cell plate maturation. Plant Physiol. 165, 1019-1034 (2014).

49. Rybak, K. et al. Plant cytokinesis is orchestrated by the sequential action of the TRAPPII and exocyst tethering complexes. Dev. Cell 29, 607-620 (2014).

50. Richter, S. et al. Polarized cell growth in Arabidopsis requires endosomal recycling mediated by GBF1-related ARF exchange factors. Nat. Cell Biol. 14, 80-86 (2011).

51. Richter, S. et al. Functional diversification of closely related ARF-GEFs in protein secretion and recycling. Nature 448, 488-492 (2007).

52. Teh, O. \& Moore, I. An ARF-GEF acting at the Golgi and in selective endocytosis in polarized plant cells. Nature 448, 493 (2007).

53. Richter, S. et al. Delivery of endocytosed proteins to the cell-division plane requires change of pathway from recycling to secretion. Elife 3, e02131 (2014).

54. Singh, M. K. \& Jürgens, G. Specificity of plant membrane trafficking-ARFs, regulators and coat proteins. Semin. Cell Dev. Biol. 80, 85-93 (2018).

55. Boutté, Y. et al. ECHIDNA-mediated post-Golgi trafficking of auxin carriers for differential cell elongation. Proc. Natl Acad. Sci. USA 110, 16259-16264 (2013).

56. Heinze, L. et al. EPSIN1 and MTV1 define functionally overlapping but molecularly distinct trans-Golgi network subdomains in Arabidopsis. Proc. Natl Acad. Sci. USA 117, 25880-25889 (2020).

57. Gendre, D. et al. Conserved Arabidopsis ECHIDNA protein mediates trans-Golgi-network trafficking and cell elongation. Proc. Natl Acad. Sci. USA 108, 8048-8053 (2011).

58. Ahmed, S. M. et al. Exocyst dynamics during vesicle tethering and fusion. Nat. Commun. 9, 5140 (2018).

59. Saeed, B., Brillada, C., Trujillo, M. Dissecting the plant exocyst. Curr Opin Plant Biol. 52, 69-76 (2019).

60. Drdová, E. J. The Secretory Vesicles Tethering Complex Exocyst and the Auxin Transport Polarization. Ph.D. thesis. (Charles University, Prague, 2017).

61. Synek, L. et al. Plasma membrane phospholipid signature recruits the plant exocyst complex via the EXO70A1 subunit. Proc Natl Acad Sci USA. 118, (2021).

62. Vermeer, J. E. M. \& Munnik, T. Using genetically encoded fluorescent reporters to image lipid signalling in living plants. Plant Lipid Signal. Protoc. 1009, 283-289 (2013).

63. Vermeer, J. E. M. et al. Imaging phosphatidylinositol 4-phosphate dynamics in living plant cells. Plant J. 57, 356-372 (2009).

64. Tan, J. Z. A. \& Gleeson, P. A. Cargo sorting at the trans-Golgi network for shunting into specific transport routes: role of Arf small G proteins and adaptor complexes. Cells 8, 531 (2019).

65. Arora, D. \& Van Damme, D. Motif-based endomembrane trafficking. Plant Physiol. 186, 221-238 (2021).

66. Pakdel, M. \& von Blume, J. Exploring new routes for secretory protein export from the trans-Golgi network. Mol. Biol. Cell 29, 235-240 (2018).

67. Kienzle, C. \& von Blume, J. Secretory cargo sorting at the trans-Golgi network. Trends Cell Biol. 24, 584-593 (2014).

68. Stoops, E. H. \& Caplan, M. J. Trafficking to the apical and basolateral membranes in polarized epithelial cells. J. Am. Soc. Nephrol. 25, 1375-1386 (2014).

69. Nemoto, K. et al. Autophosphorylation profiling of Arabidopsis protein kinases using the cell-free system. Phytochemistry 72, 1136-1144 (2011).

70. Li, C. et al. Glycosylphosphatidylinositol-anchored proteins as chaperones and co-receptors for FERONIA receptor kinase signaling in Arabidopsis. Elife 4, e06587 (2015). 
71. Bertolotti, G. et al. A PHABULOSA-controlled genetic pathway regulates ground tissue patterning in the Arabidopsis root. Curr. Biol. 31, 420-426.e6 (2021).

72. Miyashima, S. et al. A comprehensive expression analysis of the arabidopsis MICRORNA165/6 gene family during embryogenesis reveals a conserved role in meristem specification and a non-cell-autonomous function. Plant Cell Physiol. 54, 375-384 (2013).

73. Czechowski, T., Stitt, M., Altmann, T., Udvardi, M. K. \& Scheible, W.-R. Genome-wide identification and testing of superior reference genes for transcript normalization in arabidopsis. Plant Physiol. 139, 5-17 (2005).

\section{Acknowledgements}

We thank Dr. Zhenbiao Yang, and Dr. Mien Van der Ven (each from UC, Riverside) for kindly sharing materials and equipment. We thank all the members of the Van Norman lab for discussions of the project and feedback on the paper while it was in preparation. We thank Dr. Glenn Hicks and Dr. Ross Sozzani for the critical reading of our paper prior to submission. We appreciate access to and assistance from the Institute of Integrative Genome Biology Genomics Core Facility and Microscopy Core Facility (UC, Riverside). An NSF-GRFP award supported J.N.T (\#DGE-1326120) and this work was supported by Initial Complement (IC) funds from the University of California, Riverside, USDA-NIFA-CA-R-BPS-5156-H, and an NSF CAREER award \#1751385 to J.M.V.N.

\section{Author contributions}

Conceptualization: C.R-F. and J.M.V.N.; Methodology and Investigation: C.R-F., R.C., J.N.T., and J.M.V.N.; Resources: C.R-F., R.C., J.N.T., and J.M.V.N.; Writing - Original Draft: C.R.F.; Writing - Review, Editing: C.R-F., J.M.V.N., R.C, and J.N.T.; Writing Revision: C.R-F. and J.M.V.N., Visualization: C.R-F., R.C.; Supervision: J.M.V.N.; Funding Acquisition: J.M.V.N and J.N.T

\section{Competing interests}

The authors declare no competing interests.

\section{Additional information}

Supplementary information The online version contains supplementary material available at https://doi.org/10.1038/s41467-021-27913-1.

Correspondence and requests for materials should be addressed to Jaimie M. Van Norman.

Peer review information Nature Communications thanks Kimberly Gallagher and Markus Grebe for their contribution to the peer review of this work.

Reprints and permission information is available at http://www.nature.com/reprints

Publisher's note Springer Nature remains neutral with regard to jurisdictional claims in published maps and institutional affiliations.

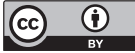

Open Access This article is licensed under a Creative Commons Attribution 4.0 International License, which permits use, sharing, adaptation, distribution and reproduction in any medium or format, as long as you give appropriate credit to the original author(s) and the source, provide a link to the Creative Commons license, and indicate if changes were made. The images or other third party material in this article are included in the article's Creative Commons license, unless indicated otherwise in a credit line to the material. If material is not included in the article's Creative Commons license and your intended use is not permitted by statutory regulation or exceeds the permitted use, you will need to obtain permission directly from the copyright holder. To view a copy of this license, visit http://creativecommons.org/ licenses/by/4.0/.

(C) The Author(s) 2022 